\title{
ACCURATE SOLUTIONS FOR RADIATIVE HEAT TRANSFER IN TWO-DIMENSIONAL AXISYMMETRIC ENCLOSURES WITH GAS RADIATION AND REFLECTIVE SURFACES
}

\section{P. Perez and M. El Hafi}

École des Mines d'Albi-Carmaux, Campus Jarlard, Albi, France

\section{P. J. Coelho}

Instituto Superior Técnico, Mechanical Engineering Department, Lisboa, Portugal

\section{R. Fournier}

Laboratoire d'Énergétique, U. Paul Sabatier,

Toulouse, France

\begin{abstract}
Accurate solutions for benchmarking purposes in two-dimensional axisymmetric enclosures with reflective surfaces have been obtained using the Monte Carlo method (MCM) based on the net exchange formulation (NEF). Previous applications of the MCM-NEF have been restricted to multidimensional problems with black boundaries or one-dimensional problems with gray boundaries. Here, the extension to multidimensional enclosures with gray boundaries is presented. The medium is a mixture of $\mathrm{H}_{2} \mathrm{O}, \mathrm{CO}_{2}, \mathrm{~N}_{2}$, and soot at atmospheric pressure, and its radiative properties are computed using the correlated $k$-distribution method. Predictions obtained using the discrete ordinates method are included, showing good agreement with the benchmark MCM/NEF solutions.
\end{abstract}

\section{INTRODUCTION}

In these last decades, numerous authors have pointed out the importance of radiative heat transfer in combustion systems such as diesel engines, boilers, furnaces, rocket engines, and many other practical applications which involve high temperatures and large scales $[1,2]$. Only a few analytical or quasi-exact solutions are available in the literature for simple geometries such as two-dimensional rectangular/axisymmetrical and three-dimensional rectangular enclosures with gray media. Accurate results of multidimensional nongray radiation analysis of real gases is typically lacking. That is due mainly to the unacceptable computing time required 


\begin{tabular}{|c|c|c|c|}
\hline \multicolumn{4}{|c|}{ NOMENCLATURE } \\
\hline$A$ & area of cell faces (DOM) & $\beta$ & direction cosine \\
\hline$f_{v}$ & soot volumetric fraction & $\Gamma$ & visibility factor \\
\hline $\mathcal{F}$ & directional probability density & $\delta$ & Dirac function \\
\hline & function for reflexion & $\epsilon$ & accuracy \\
\hline$g$ & cumulative distribution function & $\varepsilon$ & emissivity \\
\hline & of the absorption coefficient & $\eta$ & wave number \\
\hline$I_{\eta}$ & spectral radiation intensity & $\theta$ & angle between direction $\mathrm{u}$ and \\
\hline$L$ & length & & normal to a surface \\
\hline$M$ & number of directions & $\kappa$ & absorption coefficient \\
\hline$N_{b}$ & number of narrow bands & $\mu$ & direction cosine \\
\hline$N_{Q}$ & number of quadrature points & $\xi$ & direction cosine \\
\hline & (CK method) & $\sigma$ & length \\
\hline $\begin{array}{l}N_{s} \\
N_{v}\end{array}$ & $\begin{array}{l}\text { number of surfaces } \\
\text { number of volumes }\end{array}$ & $\tau_{i j}$ & $\begin{array}{l}\text { spectral transmissivity from point } P_{i} \\
\text { to point } P_{j}\end{array}$ \\
\hline $\begin{array}{l}p_{n} \\
\mathbf{q}\end{array}$ & $\begin{array}{l}\text { probability of band number } n \\
\text { heat flux vector }\end{array}$ & $\varphi_{\left(S_{i}, S_{j}\right)}$ & $\begin{array}{l}\text { net radiative exchange between } \\
\text { surfaces } S_{i} \text { and } S_{j}\end{array}$ \\
\hline$r$ & radial coordinate & $\varphi_{\left(S_{i}, V_{j}\right)}$ & net radiative exchange between \\
\hline $\mathbf{r}$ & position vector & & surface $S_{i}$ and volume $V_{j}$ \\
\hline$R$ & radius & $\varphi_{\left(V_{i}, S_{j}\right)}$ & net radiative exchange between \\
\hline$S$ & surface & & volume $V_{i}$ and surface $S_{j}$ \\
\hline $\mathbf{u}$ & direction vector & $\varphi_{\left(V_{i}, V_{j}\right)}$ & net radiative exchange between \\
\hline$V$ & volume or cell volume & & volumes $V_{i}$ and $V_{j}$ \\
\hline$w_{m}$ & DOM quadrature weight for & $\omega$ & solid angle \\
\hline$w_{k}$ & $\begin{array}{l}\text { direction } \mathrm{m} \\
\text { DOM quadrature weight for } \mathrm{CK}\end{array}$ & Subscripts & \\
\hline & method & $w$ & wall \\
\hline $\begin{array}{l}W \\
x\end{array}$ & $\begin{array}{l}\text { Monte Carlo integration weight } \\
\text { axial coordinate }\end{array}$ & Superscripts & \\
\hline$\alpha$ & absorptivity & $m$ & direction \\
\hline$\alpha_{m}$ & curvature coefficient of angular & 0 & blackbody \\
\hline & redistribution $(\mathrm{DOM})$ & - & mean value \\
\hline
\end{tabular}

by the detailed models of gaseous radiation calculations in multiple dimensions. Considering this state of the art, a first benchmark numerical solution has been proposed recently by Coelho et al. [3], for two-dimensional black enclosures with nongray sooting media. The aim of the present article is to extend this work to enclosures bounded by gray diffuse and specular walls. When using the Monte Carlo method (MCM) with a net exchange formulation (NEF), it is not possible any more to rely on the physical pictures of photon statistical transport to derive the algorithm. It is required that the algorithm be rigorously justified by a complete formulation work. This was done only with reflection in [4] for 1-D geometries, in a way that could not be simply extended to complex geometries. This is therefore the first time that the extension of a net-exchange MCM to reflection in any geometry is presented for publication. A new formulation of the Monte Carlo-net exchange formulation (MCM-NEF) involving diffuse and specular walls is detailed in the section devoted to theoretical methods. The results presented in the last section are useful in evaluating the accuracy of other approximate numerical methods such as the discrete ordinates method (DOM), which remains adequate for most practical applications [5-7]. 


\section{THEORETICAL METHODS}

\subsection{The Net Exchange Formulation and the Monte Carlo Method}

The many variants of the Monte Carlo method are generally recognized as accurate solution methods (see, for instance, the review of J. R. Howell in [8]), and are commonly used for benchmark purposes. Most of the techniques are presented as strict numerical implementations of photon transport stochastic models and optimization efforts essentially concern the adaptation of the random sampling procedures $[9,10]$. However, these techniques based on standard photon transport formulations frequently appear to be not efficient and to require very large numbers of statistical realizations for accurate solutions, when optical thicknesses are high, when configurations include hot spots, or, at the opposite, when temperature differences are small. Recently, numerous research efforts have been undertaken to overcome these difficulties on the basis of optimized formulations [8, 11-17]. Among such optimization techniques, the MCM-NEF was shown to have many advantages in terms of statistical convergence, but a detailed formulation procedure is strictly required for each extension to new application fields $[4,16,18]$. The net exchange formulation was originally proposed in [19] for monochromatic radiative exchanges in the atmosphere, and later extended in [20] to narrow bands. It has some similarities to the zone method, but it does not require that the surface and volume zones be isothermal. In the field of thermal radiation, the Monte Carlo-net exchange formulation method was first applied to one-dimensional enclosures in [14]. In this method, the net radiative heat exchanges between every volume-volume, surfacevolume, and surface-surface pair are calculated using the Monte Carlo method for the computation of the integrals that appear in the definition of the net radiative exchanges. One of the advantages of the MCM-NEF over the traditional MCM is that it intrinsically satisfies the reciprocity principle and the energy conservation, which allows one to bypass convergence difficulties in the quasi-isothermal limit and in the optically thick limit [15]. The method was extended to two-dimensional Cartesian enclosures with black walls in [16] and to one-dimensional enclosures with reflective surfaces in [4]. A very recent work using this methodology to address optically thick scattering was carried out by Eymet et al. in [18, 21]. Theoretical derivations are presented in Sections 2.1.1, 2.1.2, and 2.1.3 that allow MCM-NEF applications to multidimensional enclosures with reflective surfaces.

2.1.1. The mathematical formulation. Let $P_{i}$ be a point within volume $V_{i}$, and $Q_{1}$ be a point on surface $S_{i}$ defined respectively by the position vectors $\mathbf{r}_{P_{i}}$ and $r_{Q_{1}}$ (Figure 1). The net radiative exchange between two volumes $V_{i}$ and $V_{j}, \varphi_{\left(V_{i}, V_{j}\right)}$, or a volume $V_{i}$ and a surface $S_{j}, \varphi_{\left(V_{i}, S_{j}\right)}$, or a surface $S_{i}$ and a volume $V_{j}, \varphi_{\left(S_{i}, V_{i}\right)}$, or a surface $S_{i}$ and a surface $S_{j}, \varphi_{\left(S_{i}, S_{j}\right)}$, are expressed as follows for black walls and nonscattering media:

$$
\begin{gathered}
\varphi_{\left(V_{i}, V_{j}\right)}=\int_{0}^{\infty} d \eta \int_{V_{i}} d V_{i} \int_{V_{j}} d V_{j} \frac{\kappa_{i} \tau_{i j} \kappa_{j}}{\sigma_{i j}^{2}} \Delta I_{i j} \\
\varphi_{\left(V_{i}, S_{j}\right)}=\int_{0}^{\infty} d \eta \int_{V_{i}} d V_{i} \int_{S_{j}} d S_{j} \frac{\kappa_{i} \tau_{i j}}{\sigma_{i j}^{2}} \Delta I_{i j}
\end{gathered}
$$




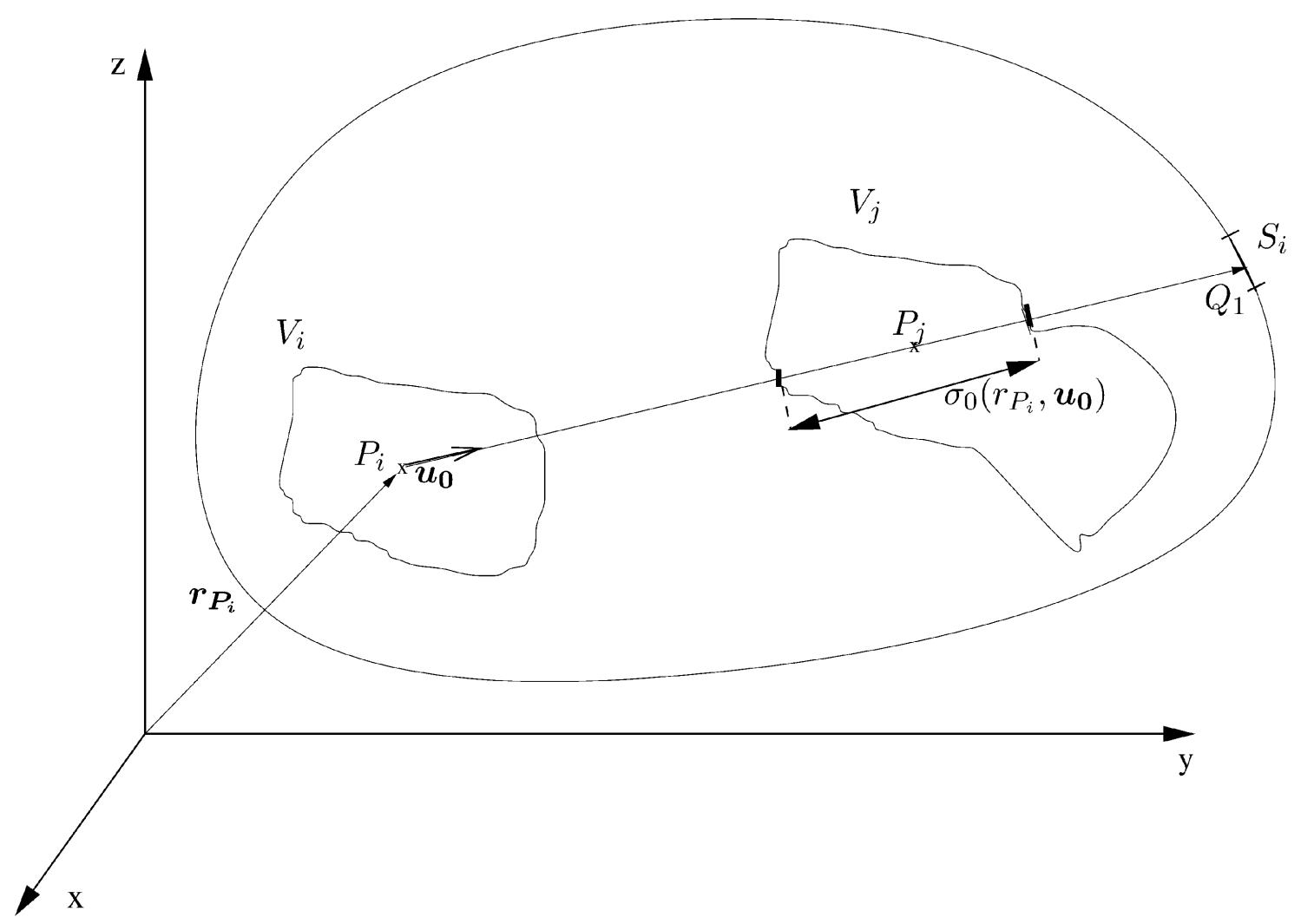

Figure 1. Exchange between two volumes for the case of black enclosures.

$$
\begin{aligned}
\varphi_{\left(S_{i}, V_{j}\right)} & =\int_{0}^{\infty} d \eta \int_{S_{i}} d S_{i} \int_{V_{j}} d V_{j} \frac{\cos \theta_{i} \tau_{i j} \kappa_{j}}{\sigma_{i j}^{2}} \Delta I_{i j} \\
\varphi_{\left(S_{i}, S_{j}\right)} & =\int_{0}^{\infty} d \eta \int_{S_{i}} d S_{i} \int_{S_{j}} d S_{j} \frac{\cos \theta_{i} \tau_{i j}}{\sigma_{i j}^{2}} \Delta I_{i j}
\end{aligned}
$$

A shortened notation is adopted for the optical properties $\kappa_{i}, \kappa_{j}, \tau_{i j}$, where $\left[\kappa_{i} \tau_{i j} \kappa_{j}\right]$ means $\left[\kappa_{\eta}\left(\mathbf{r}_{P_{i}}\right) \tau_{\eta}\left(\mathbf{r}_{p_{i}} \rightarrow \mathbf{r}_{P_{j}}\right) \kappa_{\eta}\left(\mathbf{r}_{P_{j}}\right)\right]$. The Planck function $\Delta I_{i j}$ here is the difference of the black body function taken at each point [for instance, $\left.\Delta I_{i j}=I_{\eta}^{0}\left(\mathbf{r}_{P_{i}}\right)-I_{\eta}^{0}\left(\mathbf{r}_{P_{j}}\right)\right] . \sigma_{i j}$ is the distance between point $P_{i}$ and point $P_{j}$.

Considering two volumes $V_{i}$ and $V_{j}$, the net radiative exchange between them is expressed as a multiple integral on the wave number $\eta$ and on the considered volumes. In the Monte Carlo method, the integrations are performed by generating a large number of rays, and each ray is defined by a frequency, a first exchange point $P_{i}$, and a direction $\mathbf{u}_{0}$ supporting a solid angle $\omega_{0}$. This is accomplished in terms of formulation by simple variable substitutions transforming, for instance, a volume integration over $V_{j}$ into a double integral over solid angle and over the intersecting segment (see Figure 1).

$$
\varphi_{\left(V_{i}, V_{j}\right)}=\int_{0}^{\infty} d \eta \int_{V_{i}} d V_{i} \int_{4 \pi} d \omega_{0}\left[\Gamma_{j}\left(\mathbf{r}_{P_{i}}, \mathbf{u}_{0}\right) \int_{\sigma_{0}\left(\mathbf{r}_{P_{i}}, \mathbf{u}_{0}\right)} d \sigma \kappa_{i} \tau_{i j} \kappa_{j} \Delta I_{i j}\right]
$$




$$
\begin{gathered}
\varphi_{\left(V_{i}, S_{j}\right)}=\int_{0}^{\infty} d \eta \int_{V_{i}} d V_{i} \int_{4 \pi} d \omega_{0}\left[\Gamma_{j}\left(\mathbf{r}_{P_{i}}, \mathbf{u}_{0}\right) \kappa_{i} \tau_{i j} \Delta I_{i j}\right] \\
\varphi_{\left(S_{i}, V_{j}\right)}=\int_{0}^{\infty} d \eta \int_{S_{i}} d S_{i} \int_{2 \pi} d \omega_{0}\left[\Gamma_{j}\left(\mathbf{r}_{P_{i}}, \mathbf{u}_{0}\right) \int_{\sigma_{0}\left(\mathbf{r}_{i}, \mathbf{u}\right)} d \sigma \cos \theta_{i} \tau_{i j} \kappa_{j} \Delta I_{i j}\right] \\
\varphi_{\left(S_{i}, S_{j}\right)}=\int_{0}^{\infty} d \eta \int_{S_{i}} d S_{i} \int_{2 \pi} d \omega_{0}\left[\Gamma_{j}\left(\mathbf{r}_{P_{i}}, \mathbf{u}_{0}\right) \cos \theta_{i} \tau_{i j} \Delta I_{i j}\right]
\end{gathered}
$$

For all the exchanges, $\Gamma_{j}\left(\mathbf{r}_{P_{i}}, \mathbf{u}_{0}\right)=1$ if the optical path intersects the exchange area $j$ (surface $S_{j}$ or volume $V_{j}$ ) and $\Gamma_{j}\left(\mathbf{r}_{P_{i}}, \mathbf{u}_{0}\right)=0$ if not. At this step, still considering an exchange between $V_{i}$ and $V_{j}$, the volume $V_{j}$ may be intercepted by the generated ray, defining a segment $\sigma_{0}\left(\mathbf{r}_{P_{i}}, \mathbf{u}_{0}\right)$ inside $V_{j}$. The second exchange point is then chosen on this segment at curvilinear abscissa $\sigma$ and the exchange is able to be estimated for this ray.

The net radiative heat flux on a surface $S_{i}$ is then obtained by summing all radiative exchanges between surface $S_{i}$ and all other surfaces and volumes of the system:

$$
q_{w, \text { net }, i}=\sum_{j=1}^{N_{v}} \varphi_{\left(S_{i}, V_{j}\right)}+\sum_{j=1}^{N_{s}} \varphi_{\left(S_{i}, S_{j}\right)}
$$

The radiative heat source for volume $V_{i}$ is, similarly,

$$
\int_{V_{i}} \nabla \cdot \mathbf{q} d V_{i}=\sum_{j=1}^{N_{v}} \varphi_{\left(V_{i}, V_{j}\right)}+\sum_{j=1}^{N_{s}} \varphi_{\left(V_{i}, S_{j}\right)}
$$

2.1.2. Generalization of the NEF to multiple reflections. Multiple reflexions are now considered in the NEF. New terms appear compared with Eqs. (5), (6), (7), and (8) in order to take into account the contribution of multiple reflexion rays in the net exchanges (Figure 2).

Exchange between two volumes. The net radiative exchange between two volumes is given by

$$
\begin{aligned}
\varphi_{\left(V_{i}, V_{j}\right)}= & \int_{0}^{\infty} d \eta \int_{V_{i}} d V_{i} \int_{4 \pi} d \omega_{0} \Gamma_{j}\left(\mathbf{r}_{P_{i}}, \mathbf{u}_{0}\right) \int_{\sigma_{0}\left(\mathbf{r}_{i}, \mathbf{u}_{0}\right)} d \sigma \kappa_{i} \tau_{i j} \kappa_{j} \Delta I_{i j} \\
& +\int_{2 \pi} d \omega_{1}\left\{\Gamma_{j}\left(\mathbf{r}_{Q_{1}}, \mathbf{u}_{1}\right) \int_{\sigma_{1}\left(\mathbf{r}_{Q_{1}}, \mathbf{u}_{1}\right)} d \sigma \kappa_{i} \tau_{i j} \kappa_{j} \Delta I_{i j}\left(1-\varepsilon_{1}\right) \mathcal{F}_{1}\left(\mathbf{u}_{1} \mid \mathbf{u}_{0}\right)\right. \\
& +\int_{2 \pi} d \omega_{2}\left[\Gamma_{j}\left(\mathbf{r}_{Q_{2}}, \mathbf{u}_{2}\right) \int_{\sigma_{2}\left(\mathbf{r}_{Q_{2}}, \mathbf{u}_{2}\right)} d \sigma \kappa_{i} \tau_{i j} \kappa_{j} \Delta I_{i j}\left(1-\varepsilon_{1}\right) \mathcal{F}_{1}\left(\mathbf{u}_{1} \mid \mathbf{u}_{0}\right)\right. \\
& \left.\left.\left.\times\left(1-\varepsilon_{2}\right) \mathcal{F}_{2}\left(\mathbf{u}_{2} \mid \mathbf{u}_{1}\right)+\cdots\right]\right\}\right)
\end{aligned}
$$




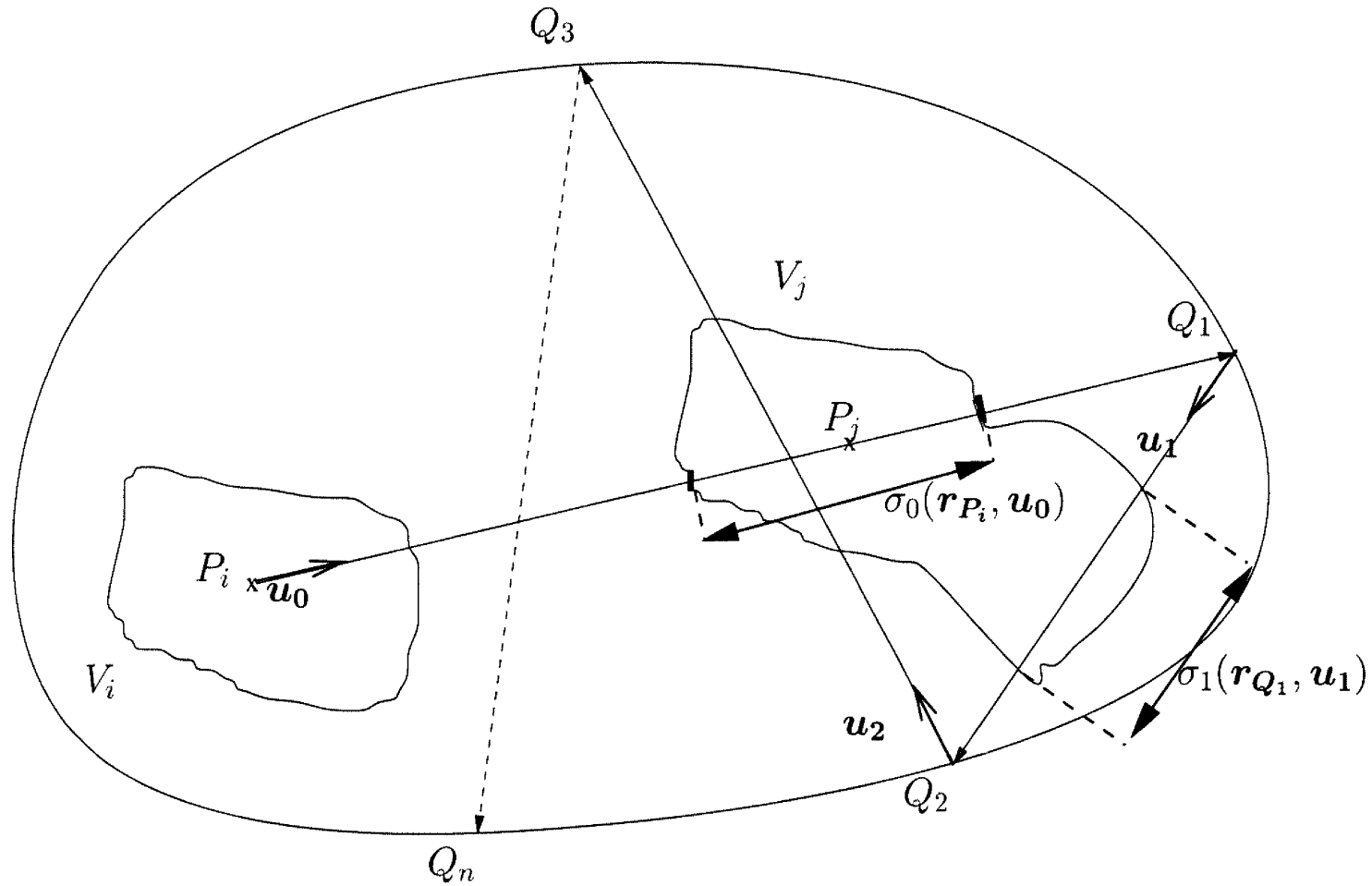

Figure 2. Exchange between two volumes for the case of reflective walls.

$\varepsilon_{1}, \varepsilon_{2}, \ldots$, stands for the directional emissivities at the reflexion points in directions $-\mathbf{u}_{0},-\mathbf{u}_{1}, \ldots$ (or the directional absorptivities in directions $+\mathbf{u}_{0},+\mathbf{u}_{1}, \ldots$ ): $\varepsilon_{1}=$ $\varepsilon\left(\mathbf{r}_{Q_{1}},-\mathbf{u}_{0}\right)=\alpha\left(\mathbf{r}_{Q_{1}}, \mathbf{u}_{0}\right), \varepsilon_{2}=\varepsilon\left(\mathbf{r}_{Q_{2}},-\mathbf{u}_{1}\right)=\alpha\left(\mathbf{r}_{Q_{2}}, \mathbf{u}_{1}\right)$.

$\mathcal{F}_{1}\left(\mathbf{u}_{1} \mid \mathbf{u}_{0}\right), \mathcal{F}_{2}\left(\mathbf{u}_{1} \mid \mathbf{u}_{2}\right)$ are the reflection-phase functions at the successive reflection points $\left[\int_{2 \pi} \mathcal{F}_{1}\left(\mathbf{u}_{1} \mid \mathbf{u}_{0}\right) d \omega_{1}=1\right]$. For a diffuse reflection, $\varepsilon_{1}\left(\mathbf{u}_{0}\right)=\varepsilon_{1}$ (independent of direction) and $\mathcal{F}_{1}\left(\mathbf{u}_{1} \mid \mathbf{u}_{0}\right)=\left[\mathbf{u}_{1} \cdot n\left(\mathbf{r}_{Q_{1}}\right)\right] / \pi$, and for a specular reflection, $\varepsilon_{1}\left(\mathbf{u}_{0}\right)=\varepsilon_{1}$ and $\mathcal{F}_{1}\left(\mathbf{u}_{1} \mid \mathbf{u}_{0}\right)=\delta\left[\mathbf{u}_{1}-\operatorname{sym}\left(-\mathbf{u}_{0}, \mathbf{r}_{Q_{1}}\right)\right]$, where $\delta$ is the Dirac function and $\operatorname{sym}\left(-\mathbf{u}_{0}, \mathbf{r}_{Q_{1}}\right)$ is the direction symmetric to $-\mathbf{u}_{0}$ relatively to the outer normal vector of the surface.

In Eq. (11), the first line is similar to the expression of $\varphi_{\left(V_{i}, V_{j}\right)}$ for black walls [Eq. (5)], and the contributions of the reflections appear on the following lines of the equation. Physically, after the first reflection at point $Q_{1}$, the ray propagates in a new direction $\mathbf{u}_{1}$, and may once again intercept the volume $V_{j}$, defining inside a new segment $\sigma_{1}\left(\mathbf{r}_{Q_{1}}, \mathbf{u}_{1}\right)$. The second line of Eq. (11) expresses the contribution of this new path to the net exchange between $V_{i}$ and $V_{j}$. Reasoning in term of NEF on the basis of this physical picture only requires considering each defined optical path as traveled both ways, from $V_{i}$ to $V_{j}$ and from $V_{j}$ to $V_{i}$ [4].

Altogether, the first line represents the contribution to $\varphi_{\left(V_{i}, V_{j}\right)}$ of all the optical paths between $V_{i}$ and $V_{j}$ without reflection. The second line represents the contribution of all the optical paths after one reflection, the third line stands for the contribution after two reflections, and so on.

The subscript $j$ is employed in $\kappa_{j}, \tau_{i j}$, and $\Delta I_{i j}$ for all the terms of Eq. (11), in each integral, but the reader should be aware that the meanings are different after 
each reflection because the corresponding variables are taken at different points in different optical paths.

Exchange between a volume and a surface. Physical considerations are the same as for the exchange between two volumes, except that integration over $\sigma_{0}, \sigma_{1}, \sigma_{2}$ (through the volume) is not required: exchanges occur only with surface points. The first line of Eq. (12) is similar to the equation without reflection [Eq. (6)], and supplementary contributions are expressed in the following lines, representing respectively the energy exchanged between $V_{i}$ and $S_{j}$ after one, two,..., reflections.

$$
\begin{aligned}
\varphi_{\left(V_{i}, S_{j}\right)}= & \int_{0}^{\infty} d \eta \int_{V_{i}} d V_{i} \int_{4 \pi} d \omega_{0} \quad \Gamma_{j}\left(\mathbf{r}_{P_{i}}, \mathbf{u}_{0}\right) \kappa_{i} \tau_{i j} \varepsilon_{1} \Delta I_{i j} \\
& +\int_{2 \pi} d \omega_{1}\left\{\Gamma_{j}\left(\mathbf{r}_{Q_{1}}, \mathbf{u}_{1}\right) \kappa_{i} \tau_{i j} \varepsilon_{2} \Delta I_{i j}\left(1-\varepsilon_{1}\right) \mathcal{F}_{1}\left(\mathbf{u}_{1} \mid \mathbf{u}_{0}\right)\right. \\
& +\int_{2 \pi} d \omega_{2}\left[\Gamma_{j}\left(\mathbf{r}_{Q_{2}}, \mathbf{u}_{2}\right) \kappa_{i} \tau_{i j} \varepsilon_{3} \Delta I_{i j}\left(1-\varepsilon_{1}\right) \mathcal{F}_{1}\left(\mathbf{u}_{1} \mid \mathbf{u}_{0}\right)\right. \\
& \left.\left.\left.\times\left(1-\varepsilon_{2}\right) \mathcal{F}_{2}\left(\mathbf{u}_{2} \mid \mathbf{u}_{1}\right)+\cdots\right]\right\}\right)
\end{aligned}
$$

The exchanges between a volume and a surface [Eq. (13)], and between two surfaces [Eq. (14)], given hereafter, are obtained in the same way.

Exchange between a surface and a volume

$$
\begin{aligned}
\varphi_{\left(S_{i}, V_{j}\right)}= & \int_{0}^{\infty} d \eta \int_{S_{i}} d S_{i} \int_{2 \pi} d \omega_{0} \Gamma_{j}\left(\mathbf{r}_{P_{i}}, \mathbf{u}_{0}\right) \int_{\sigma_{0}\left(\mathbf{r}_{P_{i}}, \mathbf{u}_{0}\right)} d \sigma \varepsilon_{i} \cos \theta_{i} \tau_{i j} \kappa_{j} \Delta I_{i j} \\
& +\int_{2 \pi} d \omega_{1}\left\{\Gamma_{j}\left(\mathbf{r}_{Q_{1}}, \mathbf{u}_{1}\right) \int_{\sigma_{1}\left(\mathbf{r}_{Q_{1}}, \mathbf{u}_{1}\right)} d \sigma \varepsilon_{i} \cos \theta_{i} \tau_{i j} \kappa_{j} \Delta I_{i j}\left(1-\varepsilon_{1}\right) \mathcal{F}_{1}\left(\mathbf{u}_{1} \mid \mathbf{u}_{0}\right)\right. \\
& +\int_{2 \pi} d \omega_{2}\left[\Gamma_{j}\left(\mathbf{r}_{Q_{2}}, \mathbf{u}_{2}\right) \int_{\sigma_{2}\left(\mathbf{r}_{Q_{2}}, \mathbf{u}_{2}\right)} d \sigma \varepsilon_{i} \cos \theta_{i} \tau_{i j} \kappa_{j} \Delta I_{i j}\left(1-\varepsilon_{1}\right) \mathcal{F}_{1}\left(\mathbf{u}_{1} \mid \mathbf{u}_{0}\right)\right. \\
& \left.\left.\left.\times\left(1-\varepsilon_{2}\right) \mathcal{F}_{2}\left(\mathbf{u}_{2} \mid \mathbf{u}_{1}\right)+\cdots\right]\right\}\right)
\end{aligned}
$$




\section{Exchange between two surfaces}

$$
\begin{aligned}
\varphi_{\left(S_{i}, S_{j}\right)}= & \int_{0}^{\infty} d \eta \int_{S_{i}} d S_{i} \int_{2 \pi} d \omega_{0} \quad \Gamma_{j}\left(\mathbf{r}_{P_{i}}, \mathbf{u}_{0}\right) \varepsilon_{i} \cos \theta_{i} \tau_{i j} \varepsilon_{1} \Delta I_{i j} \\
& +\int_{2 \pi} d \omega_{1}\left\{\Gamma_{j}\left(\mathbf{r}_{Q_{1}}, \mathbf{u}_{1}\right) \varepsilon_{i} \cos \theta_{i} \tau_{i j} \varepsilon_{2} \Delta I_{i j}\left(1-\varepsilon_{1}\right) \mathcal{F}_{1}\left(\mathbf{u}_{1} \mid \mathbf{u}_{0}\right)\right. \\
& +\int_{2 \pi} d \omega_{2}\left[\Gamma_{j}\left(\mathbf{r}_{Q_{2}}, \mathbf{u}_{2}\right) \varepsilon_{i} \cos \theta_{i} \tau_{i j} \varepsilon_{3} \Delta I_{i j}\left(1-\varepsilon_{1}\right) \mathcal{F}_{1}\left(\mathbf{u}_{1} \mid \mathbf{u}_{0}\right)\right. \\
& \left.\left.\left.\times\left(1-\varepsilon_{2}\right) \mathcal{F}_{2}\left(\mathbf{u}_{2} \mid \mathbf{u}_{1}\right)+\cdots\right]\right\}\right)
\end{aligned}
$$

The wave-number integration is carried out over narrow bands $\Delta \eta$ according to the statistical narrow band model proposed by Malkmus [22], using a $k$-distribution reformulation [23] and a correlated- $k$ assumption for the representation of inhomogeneities [24]. In the correlated- $k$ distribution approach, frequencies are rearranged and the absorption spectrum $\kappa_{\eta}$ is replaced with an equivalent spectrum $\kappa_{g}$ in which $\kappa$ is a monotonous function of a pseudo-frequency $g$. Practically, $g$ is the cumulative of the $k$-distribution and each frequential integration over a narrow band $\Delta \eta$ is transformed as $\int_{\Delta \eta} d \eta \ldots=\Delta \eta \int_{0}^{1} d g \ldots$ The shortened notation $\kappa_{i} \tau_{i j} \kappa_{j}$, for instance, now stands for $\kappa_{g}\left(\mathbf{r}_{P_{i}}\right) \tau_{g}\left(\mathbf{r}_{P_{i}} \rightarrow \mathbf{r}_{P_{j}}\right) \kappa_{g}\left(\mathbf{r}_{P_{j}}\right)$. Then, from Eqs. (11), (12), (13), and (14), it yields:

$$
\begin{aligned}
\varphi_{\left(V_{i}, V_{j}\right)} & \sum_{n=1}^{N_{b}} \Delta \eta_{n} \int_{0}^{1} d g \int_{V_{i}} d V_{i} \int_{4 \pi} d \omega_{0} \Gamma_{j}\left(\mathbf{r}_{P_{i}}, \mathbf{u}_{0}\right) \int_{\sigma_{0}\left(\mathbf{r}_{i}, \mathbf{u}_{0}\right)} d \sigma \kappa_{i} \tau_{i j} \kappa_{j} \Delta I_{i j} \\
& +\int_{2 \pi} d \omega_{1}\left\{\Gamma_{j}\left(\mathbf{r}_{Q_{1}}, \mathbf{u}_{1}\right) \int_{\sigma_{1}\left(\mathbf{r}_{Q_{1}}, \mathbf{u}_{1}\right)} d \sigma \kappa_{i} \tau_{i j} \kappa_{j} \Delta I_{i j}\left(1-\varepsilon_{1}\right) \mathcal{F}_{1}\left(\mathbf{u}_{1} \mid \mathbf{u}_{0}\right)\right. \\
& +\int_{2 \pi} d \omega_{2}\left[\Gamma_{j}\left(\mathbf{r}_{Q_{2}}, \mathbf{u}_{2}\right) \int_{\sigma_{2}\left(\mathbf{r}_{Q_{2}}, \mathbf{u}_{2}\right)} d \sigma \kappa_{i} \tau_{i j} \kappa_{j} \Delta I_{i j}\left(1-\varepsilon_{1}\right) \mathcal{F}_{1}\left(\mathbf{u}_{1} \mid \mathbf{u}_{0}\right)\right. \\
& \left.\left.\left.\times\left(1-\varepsilon_{2}\right) \mathcal{F}_{2}\left(\mathbf{u}_{2} \mid \mathbf{u}_{1}\right)+\cdots\right]\right\}\right)
\end{aligned}
$$

where $N_{b}$ is the total number of narrow bands. The parameters of the Malkmus model were taken from the database given in [25] for 367 narrow bands of $25 \mathrm{~cm}^{-1}$ width considering a spectral range from 150 to $9,300 \mathrm{~cm}^{-1}$. The number of narrow bands has been extended to 800 in order to take into account the whole spectral range from 0 to $20,000 \mathrm{~cm}^{-1}$ for soot radiation at high frequencies for high temperatures. 


$$
\begin{aligned}
& \varphi_{\left(V_{i}, S_{j}\right)}=\sum_{n=1}^{N_{b}} \Delta \eta_{n} \int_{0}^{1} d g \int_{V_{i}} d V_{i} \int_{4 \pi} d \omega_{0} \quad \Gamma_{j}\left(\mathbf{r}_{P_{i}}, \mathbf{u}_{0}\right) \kappa_{i} \tau_{i j} \varepsilon_{1} \Delta I_{i j} \\
& +\int_{2 \pi} d \omega_{1}\left\{\Gamma_{j}\left(\mathbf{r}_{Q_{1}}, \mathbf{u}_{1}\right) \kappa_{i} \tau_{i j} \varepsilon_{2} \Delta I_{i j}\left(1-\varepsilon_{1}\right) \mathcal{F}_{1}\left(\mathbf{u}_{1} \mid \mathbf{u}_{0}\right)\right. \\
& +\int_{2 \pi} d \omega_{2}\left[\Gamma_{j}\left(\mathbf{r}_{Q_{2}}, \mathbf{u}_{2}\right) \kappa_{i} \tau_{i j} \varepsilon_{3} \Delta I_{i j}\left(1-\varepsilon_{1}\right) \mathcal{F}_{1}\left(\mathbf{u}_{1} \mid \mathbf{u}_{0}\right)\right. \\
& \left.\left.\left.\times\left(1-\varepsilon_{2}\right) \mathcal{F}_{2}\left(\mathbf{u}_{2} \mid \mathbf{u}_{1}\right)+\cdots\right]\right\}\right) \\
& \varphi_{\left(S_{i}, V_{j}\right)}=\sum_{n=1}^{N_{b}} \Delta \eta_{n} \int_{0}^{1} d g \int_{S_{i}} d S_{i} \int_{2 \pi} d \omega_{0} \quad \Gamma_{j}\left(\mathbf{r}_{p_{i}}, \mathbf{u}_{0}\right) \int_{\sigma_{0}\left(\mathbf{r}_{i}, \mathbf{u}\right)} d \sigma \varepsilon_{i} \cos \theta_{i} \tau_{i j} \kappa_{j} \Delta I_{i j} \\
& +\int_{2 \pi} d \omega_{1}\left\{\Gamma_{j}\left(\mathbf{r}_{Q_{1}}, \mathbf{u}_{1}\right) \int_{\sigma_{1}\left(\mathbf{r}_{Q_{1}}, \mathbf{u}_{1}\right)} d \sigma \varepsilon_{i} \cos \theta_{i} \tau_{i j} \kappa_{j} \Delta I_{i j}\left(1-\varepsilon_{1}\right) \mathcal{F}_{1}\left(\mathbf{u}_{1} \mid \mathbf{u}_{0}\right)\right. \\
& +\int_{2 \pi} d \omega_{2}\left[\Gamma_{j}\left(\mathbf{r}_{Q_{2}}, \mathbf{u}_{2}\right) \int_{\sigma_{2}\left(\mathbf{r}_{Q_{2}}, \mathbf{u}_{2}\right)} d \sigma \varepsilon_{i} \cos \theta_{i} \tau_{i j} \kappa_{j} \Delta I_{i j}\left(1-\varepsilon_{1}\right) \mathcal{F}_{1}\left(\mathbf{u}_{1} \mid \mathbf{u}_{0}\right)\right. \\
& \left.\left.\left.\times\left(1-\varepsilon_{2}\right) \mathcal{F}_{2}\left(\mathbf{u}_{2} \mid \mathbf{u}_{1}\right)+\cdots\right]\right\}\right) \\
& \varphi_{\left(S_{i}, S_{j}\right)}=\sum_{n=1}^{N_{b}} \Delta \eta_{n} \int_{0}^{1} d g \int_{S_{i}} d S_{i} \int_{2 \pi} d \omega_{0} \quad \Gamma_{j}\left(\mathbf{r}_{P_{i}}, \mathbf{u}_{0}\right) \varepsilon_{i} \cos \theta_{i} \tau_{i j} \varepsilon_{1} \Delta I_{i j} \\
& +\int_{2 \pi} d \omega_{1}\left\{\Gamma_{j}\left(\mathbf{r}_{Q_{1}}, \mathbf{u}_{1}\right) \varepsilon_{i} \cos \theta_{i} \tau_{i j} \varepsilon_{2} \Delta I_{i j}\left(1-\varepsilon_{1}\right) \mathcal{F}_{1}\left(\mathbf{u}_{1} \mid \mathbf{u}_{0}\right)\right. \\
& +\int_{2 \pi} d \omega_{2}\left[\Gamma_{j}\left(\mathbf{r}_{Q_{2}}, \mathbf{u}_{2}\right) \varepsilon_{i} \cos \theta_{i} \tau_{i j} \varepsilon_{3} \Delta I_{i j}\left(1-\varepsilon_{1}\right) \mathcal{F}_{1}\left(\mathbf{u}_{1} \mid \mathbf{u}_{0}\right)\right. \\
& \left.\left.\left.\times\left(1-\varepsilon_{2}\right) \mathcal{F}_{2}\left(\mathbf{u}_{2} \mid \mathbf{u}_{1}\right)+\cdots\right]\right\}\right)
\end{aligned}
$$

2.1.3. Monte Carlo integrations. A Monte Carlo algorithm based on this formulation has been developed with the use of adapted probability density functions (pdf) in order to optimize the number of sampling events and to ensure fast convergence, especially for optically thick media [15]. For instance, if pdf functions are introduced in the net exchange between two volumes [Eq. (15)], 
one obtains:

$$
\begin{aligned}
\varphi_{\left(V_{i}, V_{j}\right)}= & \sum_{n=1}^{N_{b}} \Delta \eta_{n} p_{n} \frac{1}{p_{n}} \int_{0}^{1} \operatorname{pdf}(g) \frac{1}{\operatorname{pdf}(g)} d g \int_{V_{i}} \operatorname{pdf}\left(V_{i}\right) \frac{1}{\operatorname{pdf}\left(V_{i}\right)} d V_{i} \\
& \times \int_{4 \pi} \operatorname{pdf}\left(\omega_{0}\right) \frac{1}{\operatorname{pdf}\left(\omega_{0}\right)} d \omega_{0} \Gamma_{j}\left(\mathbf{r}_{P_{i}}, \mathbf{u}_{0}\right) \int_{\sigma_{0}\left(\mathbf{r}_{P_{i}}, \mathbf{u}_{0}\right)} \operatorname{pdf}\left(\sigma_{0}\right) \frac{1}{\operatorname{pdf}\left(\sigma_{0}\right)} d \sigma_{0} \\
& \times \kappa_{i} \tau_{i j} \kappa_{j} \Delta I_{i j} \\
& +\int_{2 \pi} \operatorname{pdf}\left(\omega_{1}\right) \frac{1}{\operatorname{pdf}\left(\omega_{1}\right)} d \omega_{1}\left\{\Gamma_{j}\left(\mathbf{r}_{Q_{1}}, \mathbf{u}_{1}\right) \int_{\sigma_{1}\left(\mathbf{r}_{Q_{1}}, \mathbf{u}_{1}\right)} \operatorname{pdf}\left(\sigma_{1}\right) \frac{1}{\operatorname{pdf}\left(\sigma_{1}\right)} d \sigma_{1}\right. \\
& \times \kappa_{i} \tau_{i j} \kappa_{j} \Delta I_{i j}\left(1-\varepsilon_{1}\right) \mathcal{F}_{1}\left(\mathbf{u}_{1} \mid \mathbf{u}_{0}\right) \\
& +\int_{2 \pi} \operatorname{pdf}\left(\omega_{2}\right) \frac{1}{\operatorname{pdf}\left(\omega_{2}\right)} d \omega_{2}\left[\Gamma_{j}\left(\mathbf{r}_{Q_{2}}, \mathbf{u}_{2}\right) \int_{\sigma_{2}\left(\mathbf{r}_{Q_{2}}, \mathbf{u}_{2}\right)} \operatorname{pdf}\left(\sigma_{2}\right) \frac{1}{\operatorname{pdf}\left(\sigma_{2}\right)} d \sigma_{2}\right. \\
& \left.\left.\left.\times \kappa_{i} \tau_{i j} \kappa_{j} \Delta I_{i j}\left(1-\varepsilon_{1}\right) \mathcal{F}_{1}\left(\mathbf{u}_{1} \mid \mathbf{u}_{0}\right)\left(1-\varepsilon_{2}\right) \mathcal{F}_{2}\left(\mathbf{u}_{2} \mid \mathbf{u}_{1}\right)+\cdots\right]\right\}\right)
\end{aligned}
$$

which may be presented as ${ }^{1}$

$$
\begin{aligned}
\varphi_{\left(V_{i}, V_{j}\right)}= & \sum_{n=1}^{N_{b}} p_{n} \Delta \eta_{n} \int_{0}^{1} \operatorname{pdf}(g) d g \int_{V_{i}} \operatorname{pdf}\left(V_{i}\right) d V_{i} \\
& \times \int_{4 \pi} \operatorname{pdf}\left(\omega_{0}\right) d \omega_{0} \int \operatorname{pdf}\left(\sigma_{0}\right) d \sigma_{0} \ldots \int_{4 \pi} \operatorname{pdf}\left(\omega_{m}\right) d \omega_{m} \int \operatorname{pdf}\left(\sigma_{m}\right) d \sigma_{m} \cdots \\
& \times\left(W_{0, V V}+W_{1, V V}+W_{2, V V}+\cdots+W_{m, V V}+\cdots\right)
\end{aligned}
$$

where $W_{m}$ represents the Monte Carlo weight corresponding to optical paths with $m$ reflections

$$
\begin{aligned}
W_{0, V V}= & \frac{1}{p_{n} \operatorname{pdf}(g) \operatorname{pdf}\left(V_{i}\right) \operatorname{pdf}\left(\omega_{0}\right)} \times \frac{\Gamma_{j} \kappa_{i} \tau_{i j} \kappa_{j} \Delta I_{i j}}{\operatorname{pdf}\left(\sigma_{0}\right)} \\
W_{1, V V}= & \frac{1}{p_{n} \quad \operatorname{pdf}(g) \operatorname{pdf}\left(V_{i}\right) \operatorname{pdf}\left(\omega_{0}\right)} \times \frac{\Gamma_{j} \kappa_{i} \tau_{i j} \kappa_{j} \Delta I_{i j}\left(1-\varepsilon_{1}\right) \mathcal{F}_{1}\left(\mathbf{u}_{1} \mid \mathbf{u}_{0}\right)}{\operatorname{pdf}\left(\sigma_{1}\right) \operatorname{pdf}\left(\omega_{1}\right)} \\
W_{2, V V}= & \frac{1}{p_{n} \quad \operatorname{pdf}(g) \operatorname{pdf}\left(V_{i}\right) \operatorname{pdf}\left(\omega_{0}\right)} \\
& \times \frac{\Gamma_{j} \kappa_{i} \tau_{i j} \kappa_{j} \Delta I_{i j}\left(1-\varepsilon_{1}\right) \mathcal{F}_{1}\left(\mathbf{u}_{1} \mid \mathbf{u}_{0}\right)\left(1-\varepsilon_{2}\right) \mathcal{F}_{2}\left(\mathbf{u}_{2} \mid \mathbf{u}_{1}\right)}{\operatorname{pdf}\left(\sigma_{2}\right) \operatorname{pdf}\left(\omega_{1}\right) \operatorname{pdf}\left(\omega_{2}\right)}
\end{aligned}
$$

${ }^{1}$ Noting that $\int_{4 \pi} \operatorname{pdf}\left(\omega_{m}\right) d \omega_{m} \equiv 1$ and $\int \operatorname{pdf}\left(\sigma_{m}\right) d \sigma_{m} \equiv 1$, which allows us to bring forward all integrals as infinite products corresponding to all potential reflection events. 


$$
\begin{aligned}
W_{m, V V}= & \frac{1}{p_{n} \quad \operatorname{pdf}(g) \operatorname{pdf}\left(V_{i}\right) \operatorname{pdf}\left(\omega_{0}\right)} \\
& \times \frac{\Gamma_{j} \kappa_{i} \tau_{i j} \kappa_{j} \Delta I_{i j}\left(1-\varepsilon_{1}\right) \mathcal{F}_{1}\left(\mathbf{u}_{1} \mid \mathbf{u}_{0}\right)\left(1-\varepsilon_{2}\right) \mathcal{F}_{2}\left(\mathbf{u}_{2} \mid \mathbf{u}_{1}\right) \cdots \mathcal{F}_{m}\left(\mathbf{u}_{m} \mid \mathbf{u}_{m-1}\right)}{\operatorname{pdf}\left(\sigma_{m}\right) \operatorname{pdf}\left(\omega_{1}\right) \operatorname{pdf}\left(\omega_{2}\right) \cdots \operatorname{pdf}\left(\omega_{m}\right)}
\end{aligned}
$$

In the same way, we can write the expression of $\varphi_{\left(V_{i}, S_{j}\right)}, \varphi_{\left(V_{i}, S_{j}\right)}, \varphi_{\left(S_{i}, S_{j}\right)}$ (see Appendix A).

Altogether, any optimized pdf set may be retained. The random sampling of each variable is performed according to this pdf set (narrow band, $g, \omega_{0}, \sigma_{0}, \omega_{1}, \sigma_{1}$, $\left.\omega_{2}, \sigma_{2}, \ldots\right)$, leading to the definition of a multiple-reflection optical path and to the successive computations of all weights $W_{0}, \ldots, W_{m}$ that are summed to obtain the total weight $W=W_{1}+W_{2}+\cdots+W_{m}+\cdots$. This procedure is reproduced for a large number of sampled optical paths and the addressed radiative quantity is estimated as the average value of all $W$. Results presented in Section 3 were obtained using the same optimized pdf set as in [15]. Statistical uncertainties are systematically associated to each result as for any Monte Carlo integration (see Appendix C).

2.1.4. Truncation error. The net radiative exchange expressions involve infinite sums over surface reflections. Practically speaking, a numerical truncation is therefore required. This truncation is performed here, as in standard MCM algorithms, on the basis of a user-defined required accuracy $\epsilon$. The multiplereflection optical path is constructed considering successive reflections, until attenuation $\tau_{i j}$ is such that contributions of all further reflections are lower than $\epsilon$. The only specific point of MCM-NEF algorithms is that when following a ray, the successive values of $\Delta I_{i j}$ (the Planck function difference that appears in $W_{0}$, $\left.W_{1}, W_{2}, \ldots\right)$ are known only when the second exchange point $\mathbf{r}_{P_{i}}$ is sampled. The truncation procedure therefore requires that an overestimate of $\Delta I_{i j}$ be introduced, which may be taken as $I_{\eta}^{0}\left(T_{\max }\right)-I_{\eta}^{0}\left(T_{\min }\right)$ where $T_{\max }$ and $T_{\min }$ are, respectively, the minimum and the maximum temperatures in the system.

\subsection{Discrete Ordinates Method}

Presently, the discrete ordinates method (DOM) [26, 27] is one of the most widely used radiation models. This is due mainly to the satisfactory accuracy of the model for most practical applications, along with its flexibility, moderate computational requirements, and simplicity of coupling with computational fluid dynamics (CFD) codes. The description of the method may be found in many publications, and therefore it is omitted here. However, the application of the DOM when the radiative properties of the medium are calculated using the CK method is much less common. This has been described in [3], and a short overview is given below for completeness.

In cylindrical coordinates $(x, r, \Psi)$, the radiative transfer equation (RTE) for an emitting, absorbing, and nonscattering medium may be written as

$$
\xi \frac{\partial I_{\eta}}{\partial x}+\frac{\beta}{r} \frac{\partial\left(r I_{\eta}\right)}{\partial r}-\frac{1}{r} \frac{\partial\left(\mu I_{\eta}\right)}{\partial \Psi}=-\kappa_{\eta} I_{\eta}+\kappa_{\eta} I_{\eta}^{0}
$$

where $\xi, \beta$, and $\mu$ are the direction cosines. 
The spatial and the angular discretizations of the RTE follow standard practices of the DOM. Hence, the spatial discretization was carried out using the finitevolume method, yielding the following discretized equation for a direction $m$ in the first quadrant (similar equations may be written for the other quadrants) and for a band of width $\Delta \eta_{l}$ :

$$
\begin{aligned}
\xi^{m} & \left.I_{i+1 / 2, j, k, l}^{m} A_{i+1 / 2, j}-I_{i-1 / 2, j, k, l}^{m} A_{i-1 / 2, j}\right) \\
& \left.+\beta^{m} \quad I_{i, j+1 / 2, k, l}^{m} A_{i, j+1 / 2}-I_{i, j-1 / 2, k, l}^{m} A_{i, j-1 / 2}\right) \\
& \left.-A_{i, j+1 / 2}-A_{i, j-1 / 2}\right)\left(\frac{\alpha_{m+1 / 2} I_{i, j, k, l}^{m+1 / 2}-\alpha_{m-1 / 2} I_{i, j, k, l}^{m-1 / 2}}{w_{m}}\right) \\
& =\left(-\kappa_{i, j, k, l} I_{i, j, k, l}^{m}+\kappa_{i, j, k, l} \bar{I}_{\eta, i, j, k, l}^{0}\right) V
\end{aligned}
$$

In this equation, the cell volume and the area of the cell faces were denoted by $V$ and $A$, respectively, $\bar{I}_{\eta}^{0}$ is the mean blackbody radiation intensity over the band, $w_{m}$ is the DOM quadrature weight, and the coefficients $\alpha$ arise from the discretization of the third term on the left of Eq. (25), as explained, e.g., in [28]. The directions $m \pm \frac{1}{2}$ define the edges of angle $\Delta \Psi$ associated with direction $m$. Subscripts $i$ and $j$ identify the control volume, and $i+\frac{1}{2}, i-\frac{1}{2}, j+\frac{1}{2}$, and $j-\frac{1}{2}$ refer to the east, west, north, and south cell faces of that control volume, respectively. Subscripts $k$ and $l$ denote the CK quadrature point and the band under consideration. In this work, the cell-face radiation intensities were calculated using the CLAM scheme [29], which is formally second-order-accurate and bounded, and a level symmetric SN quadrature.

The total radiation intensity at a control volume is obtained by adding the contributions of all the CK quadrature points in a band, all directions and all bands:

$$
I_{i, j}=\sum_{l=1}^{N_{b}} \Delta \eta_{l} \sum_{m=1}^{M} w_{j} \sum_{k=1}^{N_{Q}} \omega_{k} I_{i, j, k, l}^{m}
$$

where $\omega_{k}$ is the CK quadrature weight, $N_{b}$ is the number of bands, $M$ is the number of directions, and $N_{Q}$ is the number of quadrature points in the CK method. There are 7 quadrature points per band and per participating gas; the weighting factors $\omega_{k}$ are taken from the database of Ecole Centrale de Paris [25, 30].

The heat flux incident on the west boundary is calculated as

$$
q_{w, j}=\sum_{l=1}^{N_{b}} \Delta \eta_{l}\left[\sum_{k=1}^{N_{Q}} \omega_{k}\left(\sum_{\substack{m=1 \\\left(\xi^{m}<0\right)}}^{M} w_{m}\left|\xi^{m}\right| I_{i, j, k, l}^{m}\right)\right]
$$

Similar equations may be written for the other boundaries. The divergence of the radiative heat flux is obtained from

$$
\nabla \cdot q_{i, j}=\sum_{l=1}^{N_{b}} \Delta \eta_{l}\left[\sum_{k=1}^{N_{Q}} \omega_{k} k_{i, j, k, l}\left(4 \pi \bar{I}_{\eta, i, j, k, l}^{0}-\sum_{m=1}^{M} w_{m} I_{i, j, k, l}^{m}\right)\right]
$$




\section{RESULTS AND DISCUSSION}

\subsection{Description of the Test Cases}

Three radiative heat transfer problems in two-dimensional axisymmetric enclosures involving gas-soot mixtures at atmospheric pressure and gray diffuse reflective walls have been solved. Specular walls have also been introduced, and comparisons with diffuse walls calculations have been performed. In the first test case, a homogeneous, isothermal configuration is considered and both the radiative heat source along the centerline of the cylinder and the incident radiative heat flux on the lateral wall are computed. Test case 2 presents a homogeneous, nonisothermal configuration. The radiative heat source is calculated along the centerline of the cylinder and the net wall heat flux is calculated on the lateral wall. Spectral data for soot and gas are the same as those used in [3]. As soot particle diameters are nanometric, the scattering effect is neglected. A third test case is considered to highlight the effect of considering either diffuse or specular walls.

The level of accuracy of the CK compared to the SNB model has been discussed in detail in our previous publication [3]; errors due to the use of these two different models do not exceed 1\% Comparisons between the SNB-CK and the SNB models have already been studied in previous articles [31-34]. In the configurations that we present here (test cases 1 and 2), the SNB-CK and the SNB give solutions in very close agreement; the error between computed solutions by both methods (DOM and $\mathrm{MCM}$ ) is due mainly to the angular and spatial discretizations of the RTE.

3.1.1. Test case 1. The enclosure is a cylinder of length $L=3 \mathrm{~m}$ and radius $R=0.5 \mathrm{~m}$. The walls are gray with an emissivity $\varepsilon_{w}=0.5$, and a temperature of $800 \mathrm{~K}$. The temperature of the gas is $1,800 \mathrm{~K}$. The composition of the medium is $20 \% \mathrm{H}_{2} \mathrm{O}, 10 \% \mathrm{CO}_{2}$, and $70 \% \mathrm{~N}_{2}$ at atmospheric pressure, and the soot volumetric fraction is $f_{v}=10^{-7}$. The radiative heat source along the centerline of the cylinder is shown in Figures $3 a$ and $3 b$. The incident heat flux on the circular wall is shown in Figures $3 c$ and $3 d$. Both the MCM and the DOM results are shown. The DOM calculations have been carried out for two different grids, with $41 \times 30$ (coarse grid) and $81 \times 60$ control volumes, respectively. Two different quadratures were also employed: S8 and S16.

The radiative heat source presents a strong variation for calculation points that are very close to the cold side walls $(x=0$ and $x=\mathrm{L}$ ). Between $x \gtrsim 0.5$ and $x \lesssim 2.5 \mathrm{~m}$, the profile is roughly flat, with a heat source of $\sim 500 \mathrm{~kW} / \mathrm{m}^{3}$ at the center of the cylinder. The incident heat flux presents also a quite flat profile far from the side walls and decreases with the distance to these walls. Figure 3 shows a minor influence of the spatial discretization on the DOM results in this test case because the medium is homogeneous and isothermal. The accuracy of the DOM is improved with the refinement of the angular discretization. As an indication, the difference between DOM and MCM results was compared at the center point $(x=1.5 \mathrm{~m})$ of the cylinder. For DOM-S16 calculations, the heat source was found to be $1.38 \%$ less than the MCM results for the coarse grid (2.40\% for DOM-S8), and $1.34 \%$ less for the finer one $(2.19 \%$ for DOM-S 8$)$. Considering the wall heat flux, a difference of 


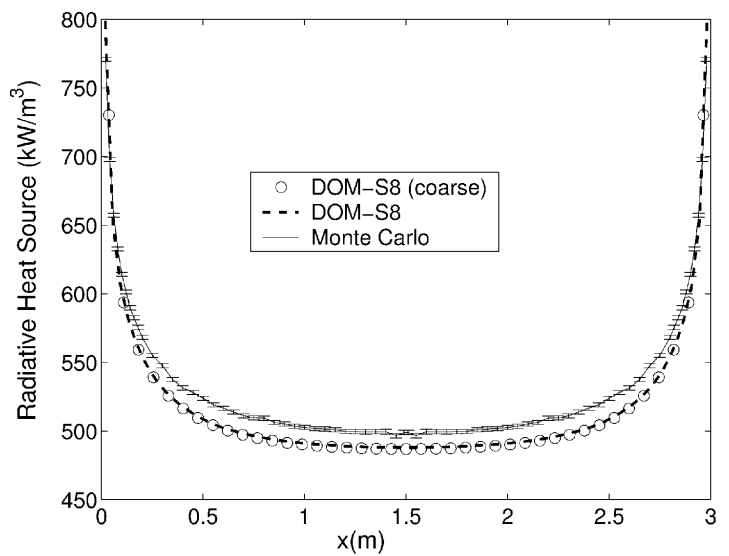

(a)

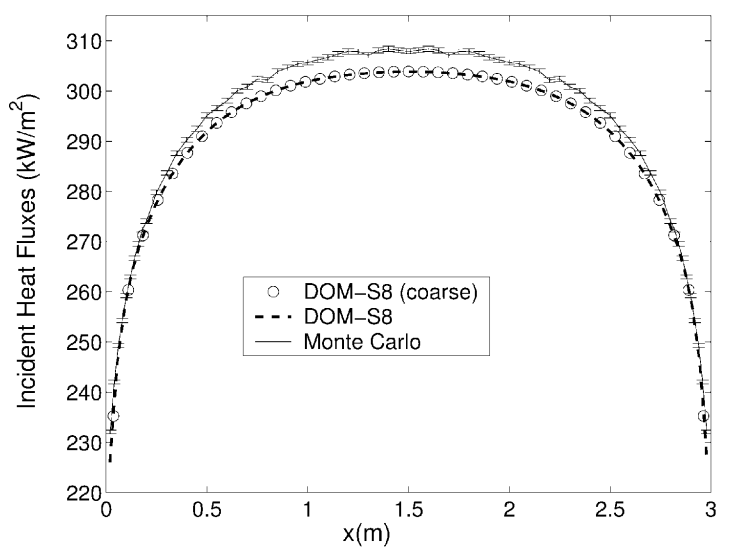

(c)

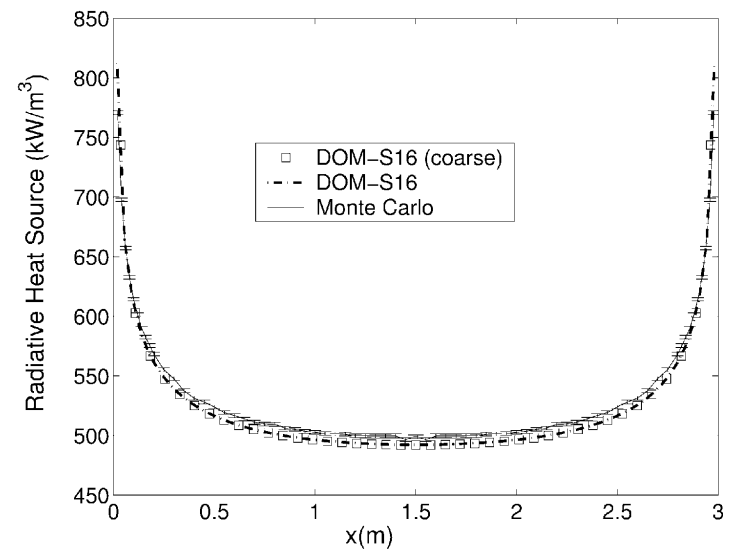

(b)

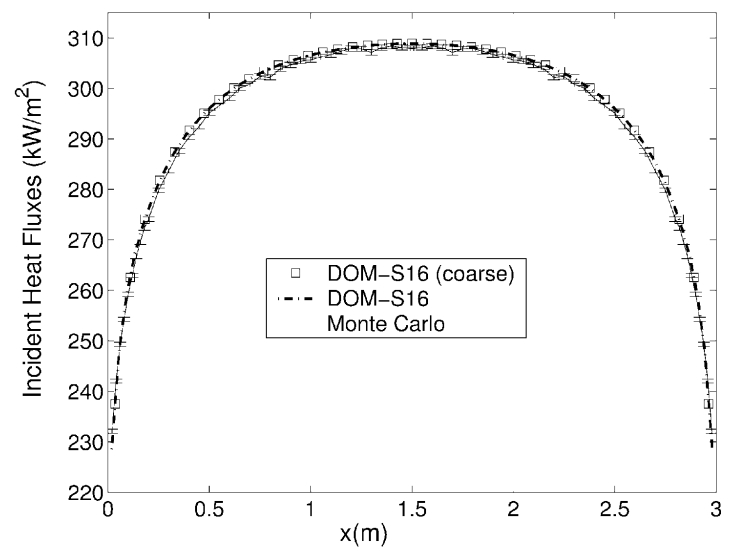

(d)

Figure 3. Heat source on the axis of a cylinder and radiative flux on the lateral side for test case 1 .

$0.32 \%$ was found between the DOM-S16 and the MCM with the coarse grid $(1.31 \%$ for DOM-S 8$)$, and a difference of $0.31 \%$ with the finer one $(1.31 \%$ for DOM-S8).

The numerical results associated to the MCM calculations can be found in Appendix B (Table 1) in order to enable readers to use them for benchmark purposes. Results of DOM calculations for specular walls are not available for this configuration, but a comparison between specular and diffuse walls is presented, using MCM calculations, in Figure 4.

3.1.2. Test case 2. An axisymmetric enclosure is again considered, in which the length of the cylinder is $L=1.2 \mathrm{~m}$ and the radius is $R=0.3 \mathrm{~m}$. The medium is homogeneous, composed of $15 \%$ water vapor and $85 \%$ nitrogen. The soot volumetric fraction is $f_{v}=10^{-6}$. The lateral wall is diffuse with an emissivity of $\varepsilon_{w}=0.8$; the side walls $(x=0$ and $x=L=1.2 \mathrm{~m})$ are black. The following twodimensional gas temperature profile is considered:

$$
T(x, r)=800+1200\left(1-\frac{r}{R}\right)\left(\frac{x}{L}\right)
$$




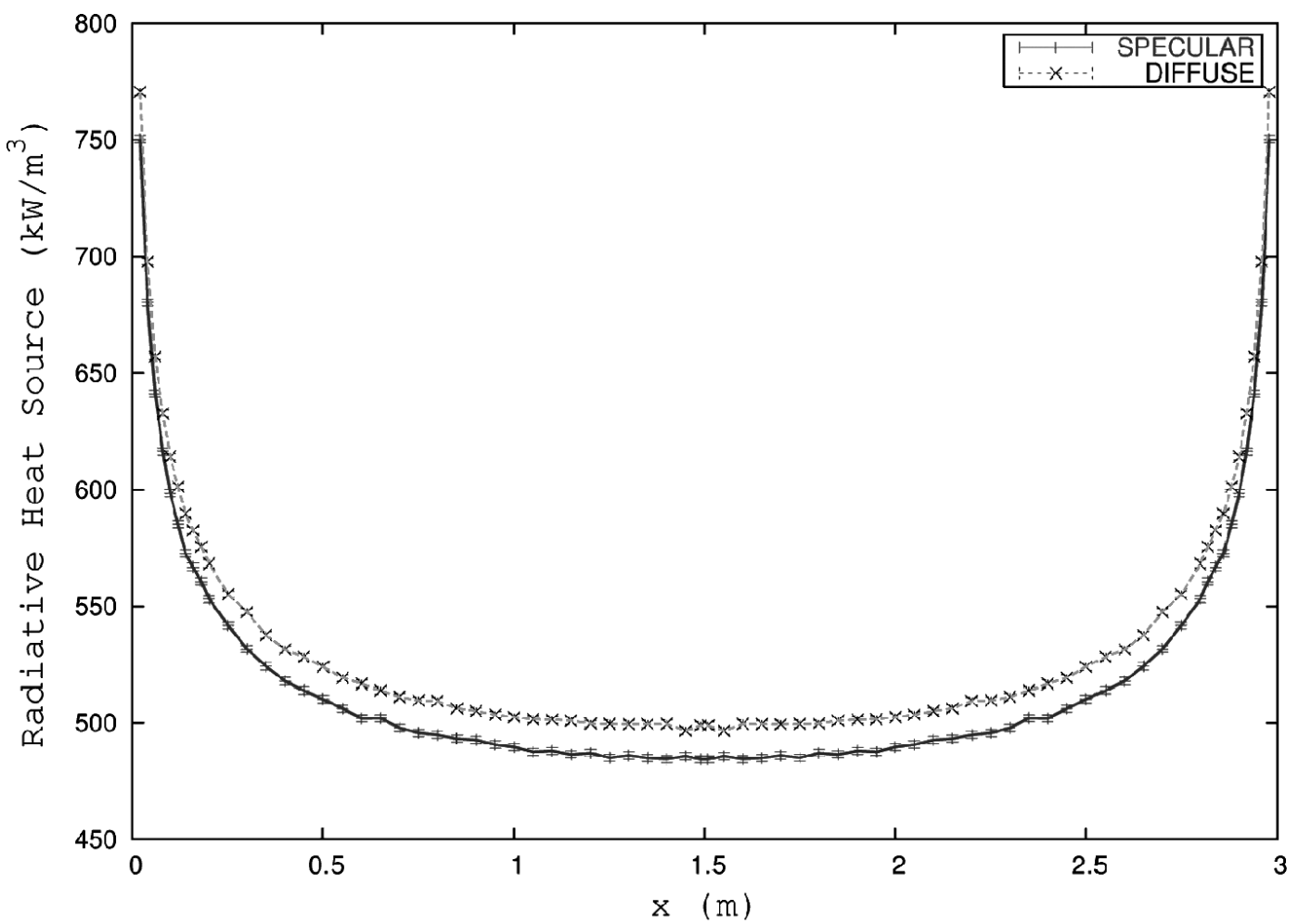

(a)

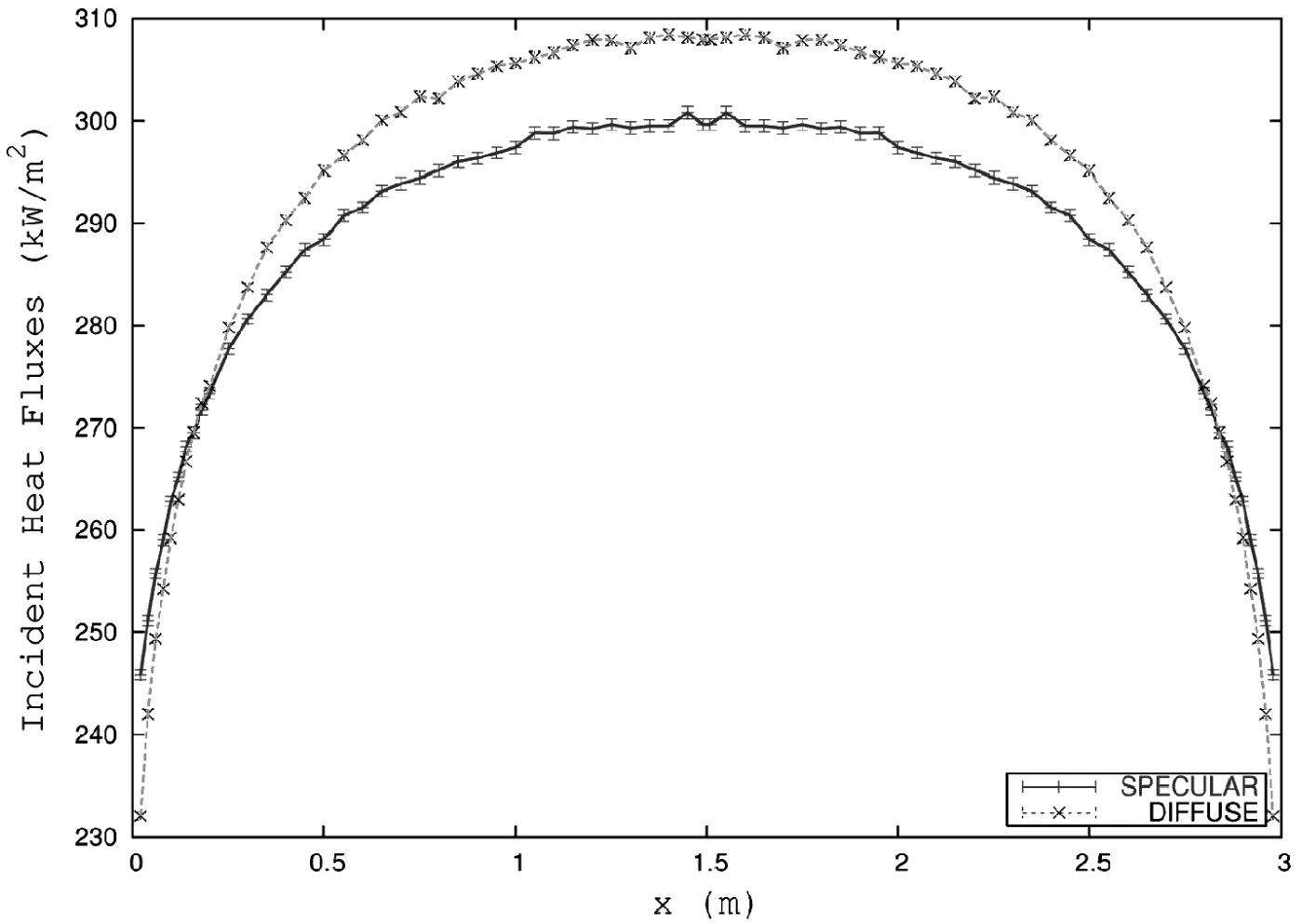

(b)

Figure 4. Comparison between a specular and a diffuse wall for test case 1 . 
This means that all the walls have a temperature of $800 \mathrm{~K}$ except the right wall $(x=L)$, which is maintained at $300 \mathrm{~K}$.

The divergence of the radiative heat flux at the centerline of the cylinder is continuously increasing with $x$, because of the temperature profile of the medium and the surrounding walls, which are maintained at the same temperature. Both the source term and the gas temperature reach the maximum at the immediate vicinity of the cold wall $(x=L)$ (Figures $5 a$ and $5 b$ ). Figures $5 c$ and $5 d$ show that the net heat flux is also increasing continuously from $x=0$ to $x \approx 1 \mathrm{~m}$, and then decreases because of the presence of the cold wall.

As for test case 1, the calculations are carried out using the DOM for two grids and two angular discretizations and are compared to the MCM results (Figure 5). The DOM results are in excellent agreement with the MCM results for the calculation of the radiative heat source on the centerline of the cylinder; the refinement of the grid seems to have no influence on the results, and the angular discretization has a minor influence. In Figure $5 c$, a discrepancy is observed between the DOM-S8 and the MCM results for the calculation of the net heat flux on the lateral wall. The discrepancy is not observed any more with a finer angular discretization, which suggests that the difference was due only to the ray

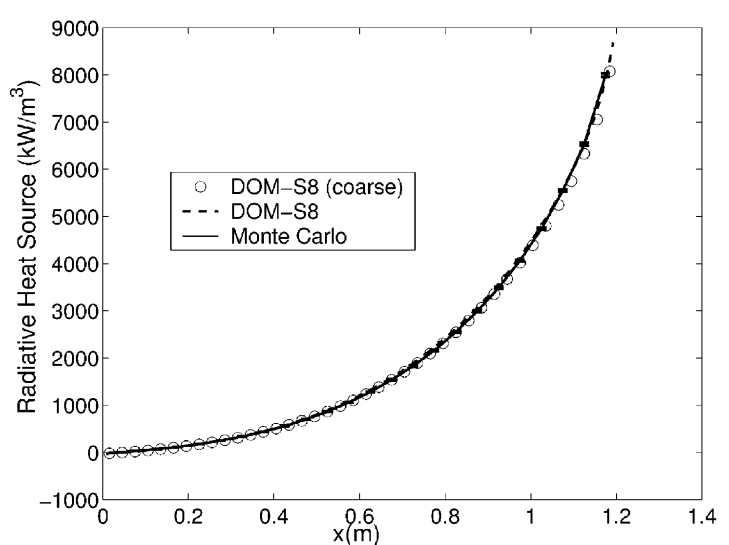

(a)

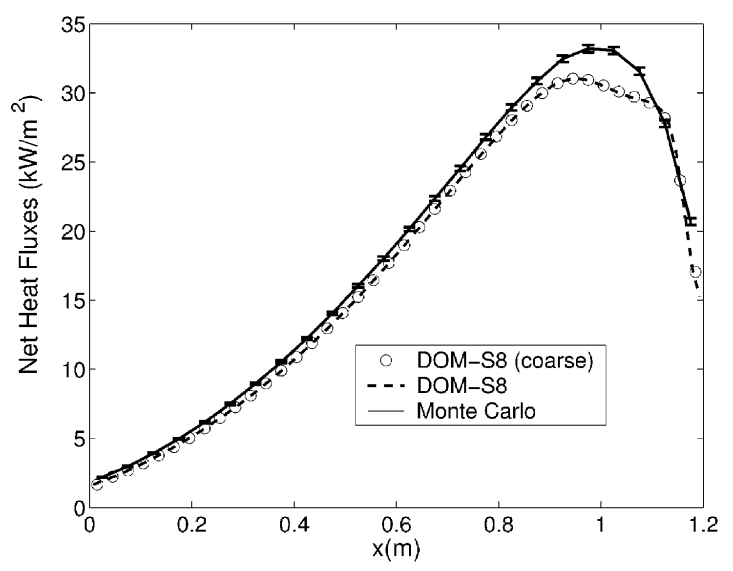

(c)

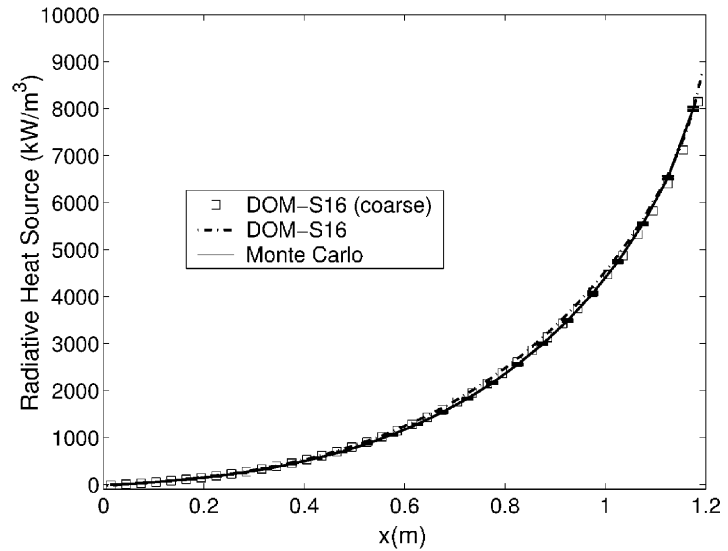

(b)

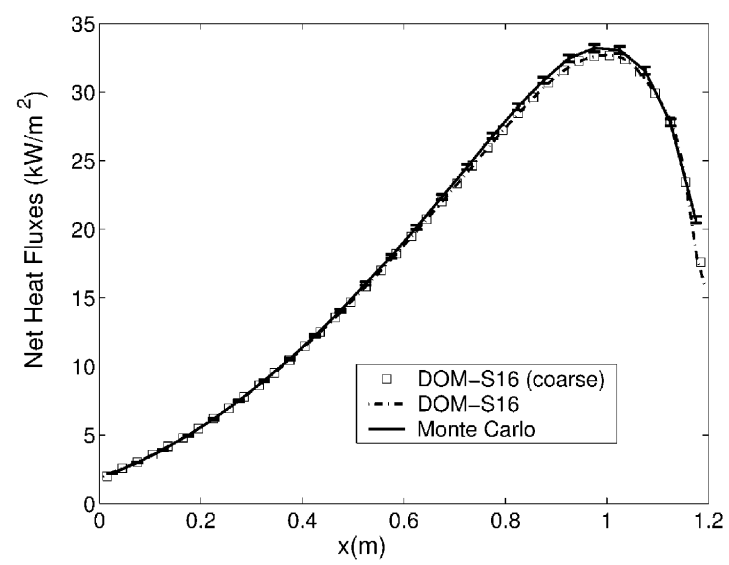

(d)

Figure 5. Heat source on the axis of a cylinder and radiative net flux on the lateral side for test case 2. 


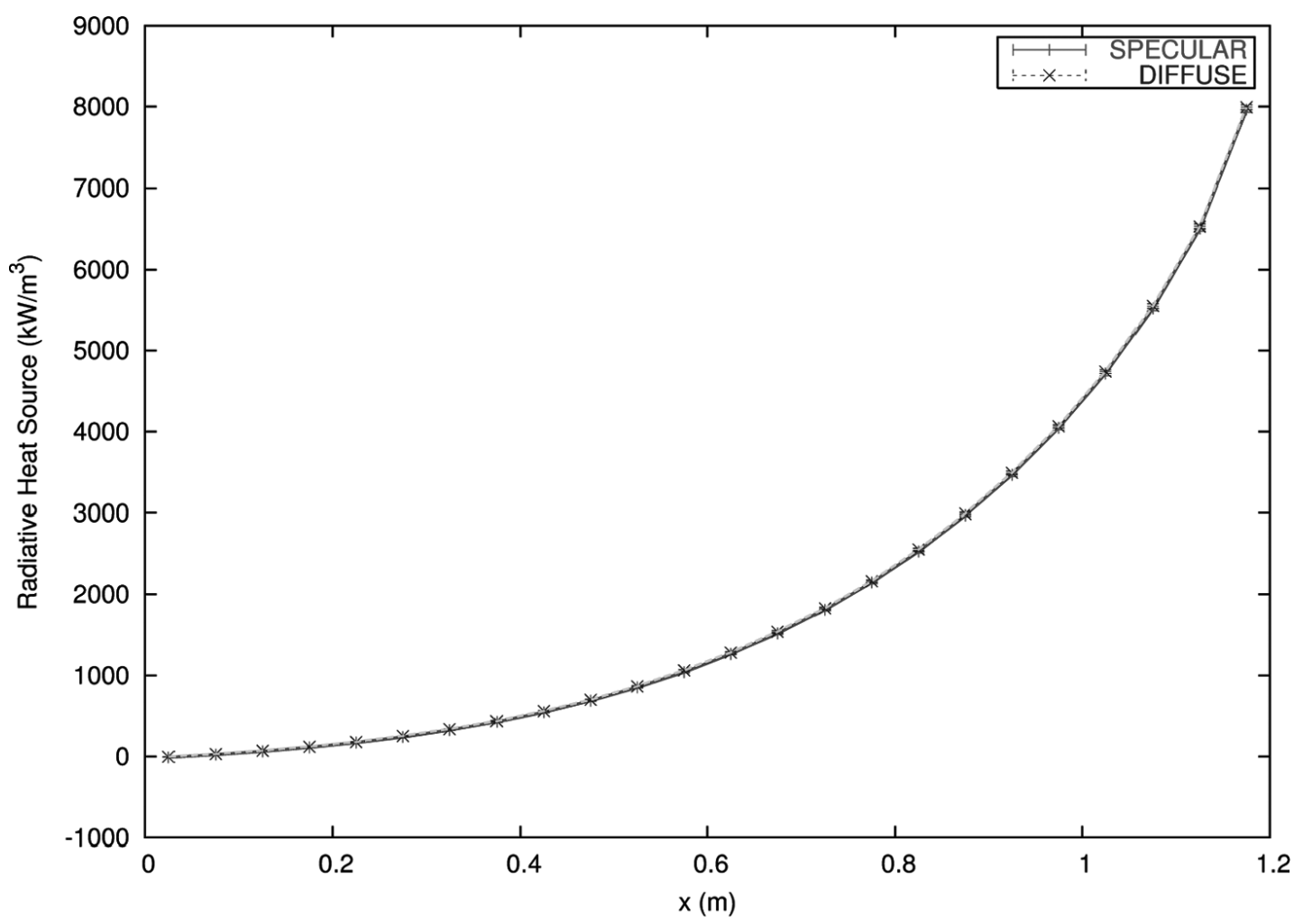

(a)

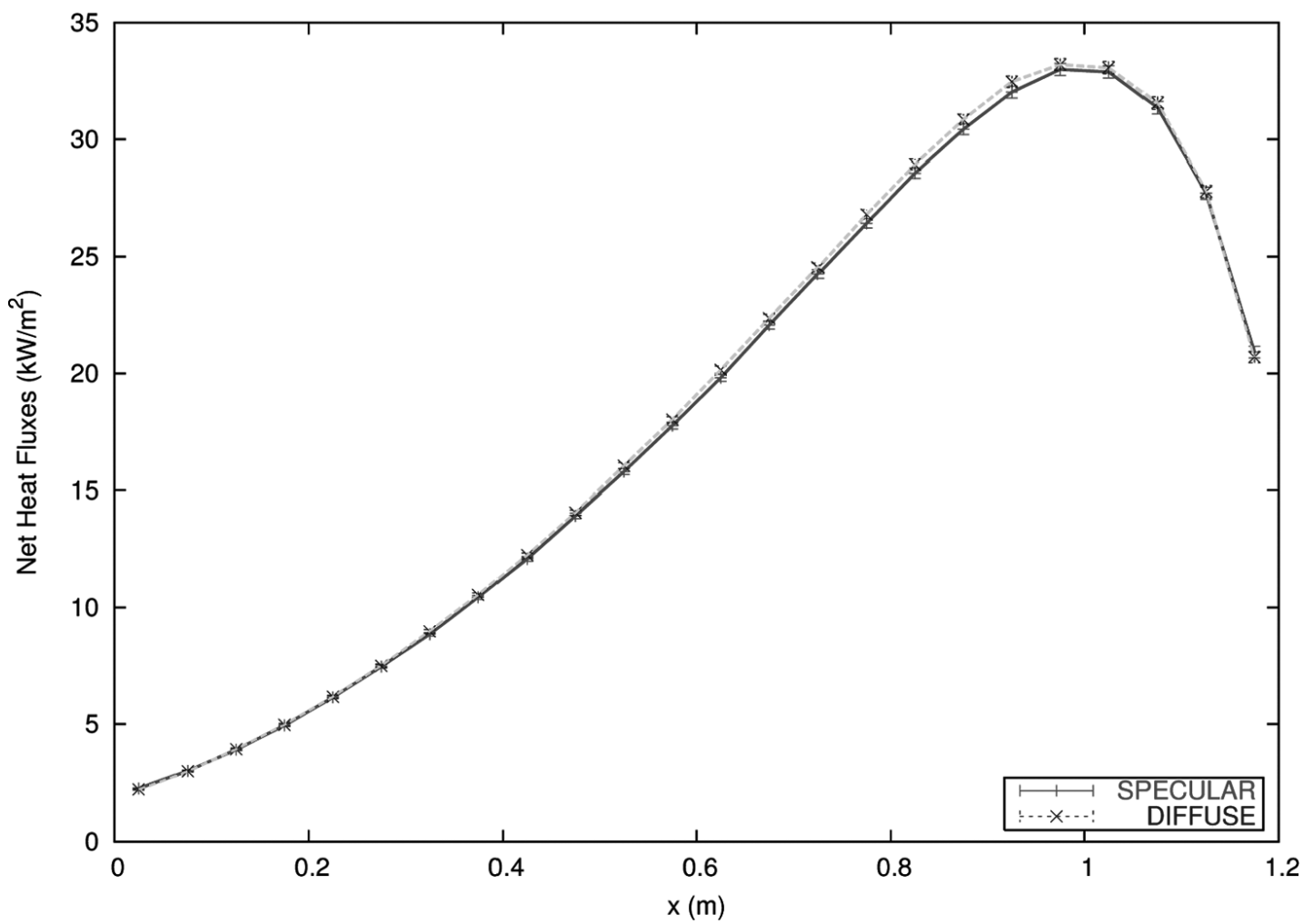

(b)

Figure 6. Comparison between a specular and a diffuse wall for test case 2 . 
effect. The MCM numerical results for test case 2 can be found in Appendix B (Table 2). The DOM (coarse-grid) numerical results are also available in Appendix B (Table 3) for some calculation points. The radiative heat source term and the net heat fluxes are computed by the MCM for diffuse and specular walls and are compared in Figure 6; the difference between the two solutions remains marginal.

3.1.3. Test case 3. The geometry (see Figure 7) is axisymmetric with a length $L=0.24 \mathrm{~m}$ and a radius $0.03 \mathrm{~m}$. The whole configuration is at a temperature of $T=1,500 \mathrm{~K}$, except the top of the cylinder at $x=0.24 \mathrm{~m}$, which is at $400 \mathrm{~K}$. The medium is a mixture of $20 \% \mathrm{H}_{2} \mathrm{O}, 10 \% \mathrm{CO}_{2}$, and a volumetric fraction of soot $f_{v}=10^{-7}$. The walls are black, except the bottom third of the cylindrical wall (from $x=0$ to $x=0.08 \mathrm{~m}$ ), which is taken either as black, diffuse, or specular $(\varepsilon=0.5)$. The average value of the heat source is computed within a cylinder of the same height (between $x=0$ and $x=0.08 \mathrm{~m}$ ) and variable radius (between $r=0$ and $r=0.03 \mathrm{~m}$ ) to show the influence of the specular reflexion (Figure 8). For black and diffuse walls, the average radiative source term is quasi-independent of the radius (within the error bars). When the radius is equal to 0.03 (radius of the cylindrical enclosure), the difference between the diffuse and specular reflexion is about $5 \%$, an order of magnitude that is comparable to the differences observed in the preceding test cases. When the radius decreases, this difference increases and reaches $20 \%$. This is due to the fact that the reflective surfaces are restricted to the bottom third of the cylindrical

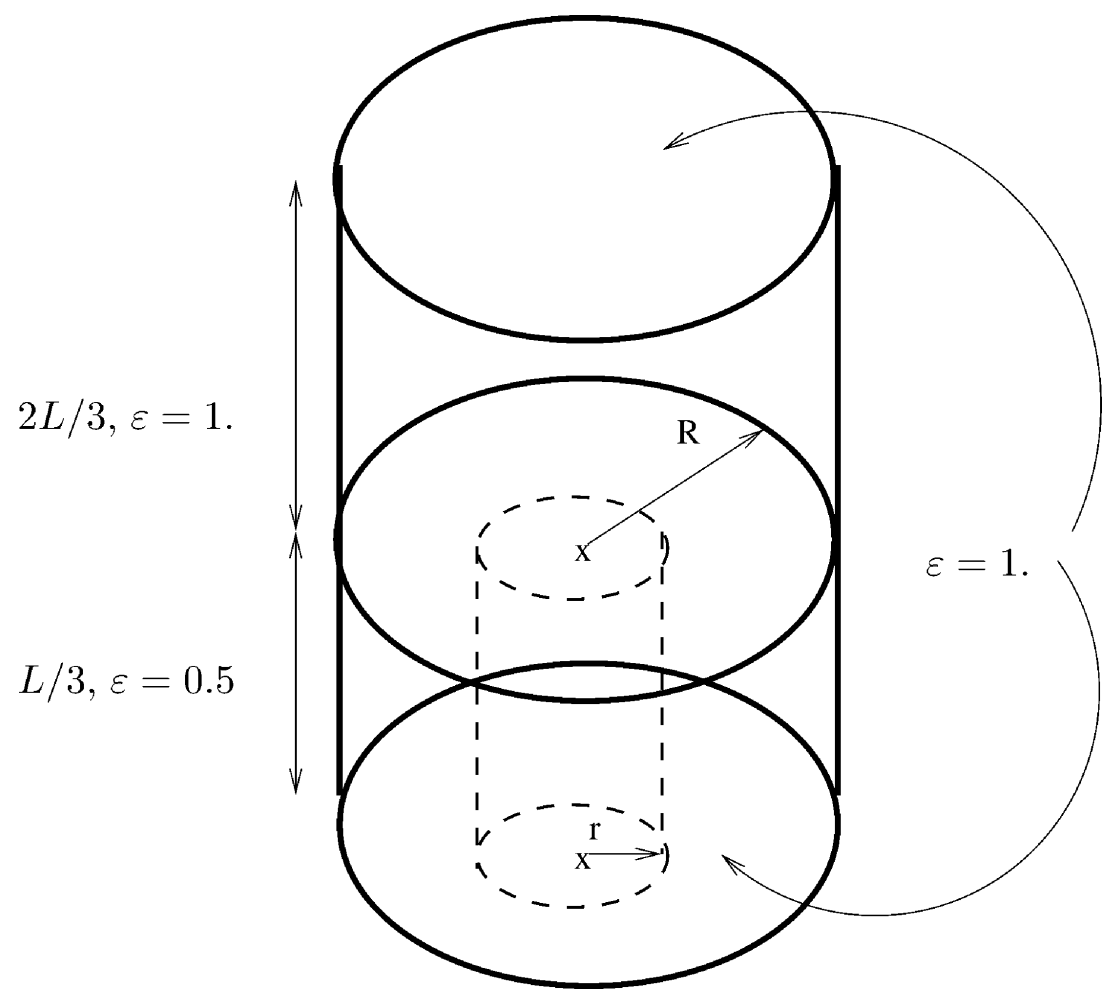

Figure 7. Average heat source term, $\int_{C}-\nabla \cdot q_{r} / \pi r^{2}(L / 3)$, computed for a cylinder with variable radius within a cylindrical enclosure. 


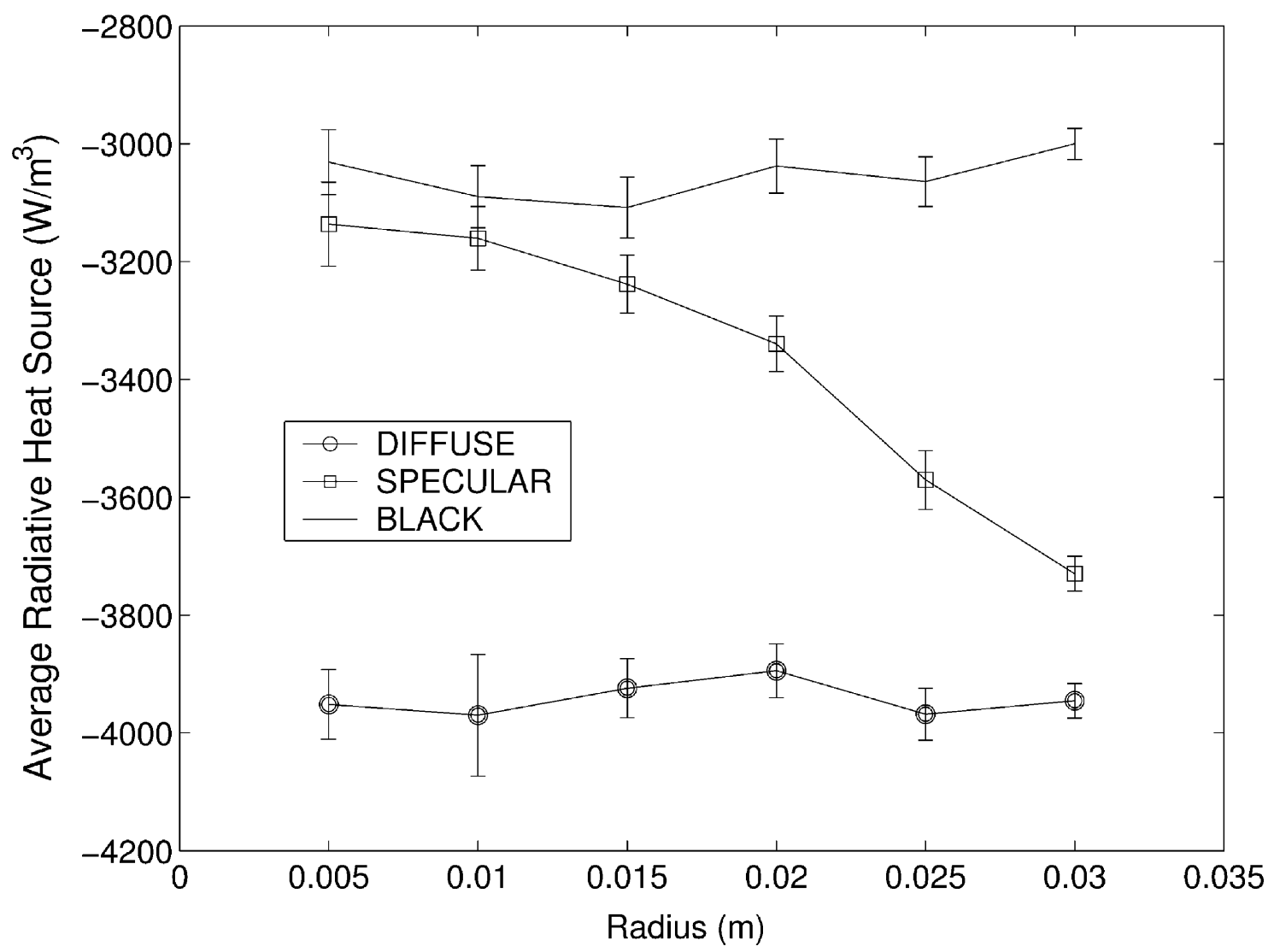

Figure 8. Schematic of test case 3 .

surface: with specular properties, no optical path can be found to reach the top cold surface with at least one reflection. The average heat source is therefore due to the direct optical paths (without reflection) only and indeed, for small radius, this average radiative source term tends to the value observed for black surfaces. This test case is therefore ideally suited to highlight the difference between specular and diffuse walls.

\section{CONCLUSION}

A Monte Carlo-net exchange formulation including multiple reflections has been detailed and used to solve two radiative heat transfer problems. For all test cases, diffuse and specular reflections were considered with a medium that is a mixture of $\mathrm{H}_{2} \mathrm{O}, \mathrm{CO}_{2}, \mathrm{~N}_{2}$, and soot, at atmospheric pressure. A discrete ordinates method has also been used on the two first test cases in order to compare with the Monte Carlo results in the case of diffuse reflexion. Altogether:

The MCM-NEF has been extended to multidimensional enclosures with reflective, diffuse, or specular boundaries.

Highly accurate solutions are available for the three configurations considered, which can be used for benchmarking. 
The DOM-CK method was shown to perform satisfactorily using the S8 quadrature (maximum error of $2.4 \%$ for radiative source term and $1.31 \%$ for radiative wall heat flux) and to yield very good predictions using S16 quadrature (maximum error of $1.38 \%$ for radiative source term and $0.32 \%$ for radiative wall heat flux).

\section{REFERENCES}

1. J. Abraham and V. Magi, Application of the Discrete Ordinates Method to Compute Radiant Heat Loss in a Diesel Engine, Numer. Heat Transfer A, vol. 31, no. 6, pp. 597610, 1997.

2. M. Mbiock and R. Weber, Radiation in Enclosures, Springer-Verlag, Berlin, 2000.

3. P. J. Coelho, P. Perez, and M. El Hafi, Benchmark Numerical Solutions for Radiative Heat Transfer in Two-Dimensional Axisymmetric Enclosures with Non-grey Sooting Media, Numer. Heat Transfer B, vol. 43, pp. 425-444, 2003.

4. M. Cherkaoui, J.-L. Dufresne, R. Fournier, J.-Y. Grandpeix, and A. Lahellec, Radiative Net-Exchange Formulation within One-Dimensional Gas Enclosures with Reflective Surfaces, J. Heat Transfer, vol. 120, pp. 275-278, 1998.

5. S. T. Thynell, Discrete-Ordinates Method in Radiative Heat Transfer, Int. J. Eng. Sci., vol. 36, pp. 1651-1675, 1998.

6. M. Sakami, A. Charette, and V. LeDez, Radiative Heat Transfer in 3-Dimensional Enclosures of Complex Geometry by Using the Discrete-Ordinate Method, J. Quant. Spectrosc. Radiative Transfer, vol. 59, pp. 117-136, 1998.

7. P. J. Coelho, Numerical Simulation of Radiative Heat Transfer from Non-gray Gases in Three-Dimensional Enclosures, J. Quant. Spectrosc. Radiative Heat Transfer, vol. 74, pp. 307-328, 2002.

8. J. R. Howell, The Monte Carlo Method in Radiative Heat Transfer, J. Heat Transfer, vol. 120 , pp. $547-560,1998$.

9. J. R. Howell, Application of the Monte Carlo Method to Heat Transfer Problems, Adv. Heat Transfer, vol. 5, pp. 1-54, 1968.

10. J. M. Hammersley and D. C. Handscomb, Monte Carlo Methods, John Wiley \& Sons, New York, 1964.

11. D. V. Walters and R. O. Buckius, Rigorous Development for Radiation Heat Transfer in Non-homogeneous Absorbing, Emitting and Scattering Media, Int. J. Heat Mass Transfer, vol. 35, pp. 3323-3333, 1992.

12. D. V. Walters and R. O. Buckius, Monte Carlo Methods for Radiative Heat Transfer in Scattering Media, Annu. Rev. Heat Transfer, vol. 5, pp. 131-176, 1994.

13. A. Ambirajan and D. C. Look, A Backward Monte Carlo Estimator for the Multiple Scattering of a Narrow Light Beam, J. Quant. Spectrosc. Radiative Heat Transfer, vol. 118 , pp. 401-407, 1996.

14. M. Cherkaoui, J.-L. Dufresne, R. Fournier, J.-Y. Grandpeix, and A. Lahellec, Monte Carlo Simulation of Radiation in Gases with a Narrow-Band Model and a Net-Exchange Formulation, J. Heat Transfer, vol. 118, pp. 401-407, 1996.

15. A. de Lataillade, J.-L. Dufresne, M. El Hafi, V. Eymet, and R. Fournier, A Net Exchange Monte Carlo Approach to Radiation in Optically Thick Systems, J. Quant. Spectrosc. Radiative Transfer, vol. 74, no. 5, pp. 563-584, 2002.

16. J.-L. Dufresne, R. Fournier, and J.-Y. Grandpeix, Méthode de Monte Carlo par échanges pour le calcul des bilans radiatifs au sein d'une cavité $2 \mathrm{~d}$ remplie de gaz, C.-R. Acad. Sci., vol. 326, pp. 33-38, 1998. 
17. L. Tessé, F. Dupoirieux, B. Zamuner, and J. Taine, Radiative Transfer in Real Gases Using Reciprocal and Forward Monte Carlo Methods and a Correlated- $K$ Approach, Int. J. Heat Mass Transfer, vol. 45, pp. 2797-2814, 2002.

18. V. Eymet, R. Fournier, S. Blanco, and J. L. Dufresne, A Boundary-Based Net Exchange Monte-Carlo Method for Absorbing and Scattering Thick Media, accepted for publication, J. Quant. Spectrosc. Radiative Transfer, 2004.

19. J. S. A. Green, Division of Radiative Streams into Internal Transfer and Cooling to Space, Quart. J. Roy. Meteorol. Soc., vol. 93, pp. 371-372, 1967.

20. J. M. Joseph and R. Bursztyn, A Radiative Cooling Model in the Thermal Infrared for Application to Models of the General Circulation, J. App. Meteorol., vol. 15, pp. 319-325, 1976.

21. V. Eymet, J. L. Dufresne, P. Riochiazzi, and R. Fournier, Longwave Radiative Analysis of Cloudy Scattering Atmospheres Using a Net Exchange Formulation, Clouds Radiation, accepted for publication, Atmospheric Research, 2004.

22. W. Malkmus, Random Lorentz Band Model with Exponential-Tailed S-1 Line-Intensity Distribution Function, J. Opt. Soc. Am., vol. 57, pp. 323-329, 1967.

23. G. A. Domoto, Frequency Integration for Radiative Transfer Problems Involving Homogeneous Non-gray Gases: The Inverse Transmission Function, J. Quant. Spectrosc. Radiative Transfer, vol. 14, pp. 935-942, 1974.

24. R. Goody and Y. Yung, Atmospheric Radiation, Oxford University Press, New York, 1989.

25. A. Soufiani and J. Taine, High Temperature Gas Radiative Property Parameters of Statistical Narrow-Band Model for $\mathrm{H}_{2} \mathrm{O}, \mathrm{CO}_{2}$ and $\mathrm{CO}$, and Correlated- $k$ Model for $\mathrm{H}_{2} \mathrm{O}$ and $\mathrm{CO}_{2}$, Int. J. Heat Mass Transfer, vol. 40, pp. 987-991, 1997.

26. S. Chandrasekar, Radiative Transfer, Dover, New York, 1960.

27. W. A. Fiveland, Discrete-Ordinates Solutions of the Radiative Transport Equation for Rectangular Enclosures, J. Heat Transfer, vol. 106, pp. 699-706, 1984.

28. W. A. Fiveland, A Discrete Ordinates Method for Prediting Radiative Heat Transfer in Axisymmetric Enclosures, ASME Paper 82-HT-20, 1982.

29. J. P. Jessee and W. A. Fiveland, Bounded, High-Resolution Differencing Schemes Applied to the Discrete Ordinates Method, J. Thermophys. Heat Transfer, vol. 11, pp. 540-548, 1997.

30. P. Rivière, D. Scutaru, A. Soufiani, and J. Taine, A New CK Data Basis Suitable from 300 to $2500 \mathrm{~K}$ for Spectrally Correlated Radiative Transfer in $\mathrm{CO}_{2}-\mathrm{H}_{2} \mathrm{O}$-Transparent Gas Mixtures, Proc. 10th Int. Heat Transfer Conf., vol. 2, pp. 129-134, 1994.

31. F. Liu, G. J. Smallwood, and O. L. Gulder, Application of the Statistical Narrow Band Correlated- $k$ Method to Low-Resolution Spectral Intensity and Radiative Heat Transfer Calculations-Effects of the Quadrature Scheme, Int. J. Heat Mass Transfer, vol. 43, pp. 3119-3135, 2000.

32. F. Liu, G. J. Smallwood, and O. L. Gulder, Application of the Statistical Narrow Band Correlated- $k$ Method to Non-grey Gas Radiation in $\mathrm{CO}_{2}-\mathrm{H}_{2} \mathrm{O}$ Mixtures: Approximate Treatments of Overlapping Bands, J. Quant. Spectrosc. Radiative Transfer, vol. 68, pp. 401-417, 2001.

33. V. Goutière, F. Liu, and A. Charette, An Assessment of Real-Gas Modelling in 2D Enclosures, J. Quant. Spectrosc. Radiative Transfer, vol. 64, pp. 299-326, 2000.

34. J. Taine and A. Soufiani, Gas IR Radiatives Properties: From Spectroscopic Data to Approximate Models, Adv. Heat Transfer, vol. 33, pp. 295-414, 1999. 


\section{APPENDIX A: WEIGHTS FOR VOLUME-SURFACE, SURFACE-VOLUME,} AND SURFACE-SURFACE EXCHANGES

$$
\begin{aligned}
& \varphi_{\left(V_{i}, S_{j}\right)}=\sum_{n=1}^{N_{b}} p_{n} \Delta_{v n} \int_{0}^{1} \operatorname{pdf}(g) d g \int_{V_{i}} \operatorname{pdf}\left(V_{i}\right) d V_{i} \\
& \int_{4 \pi} \operatorname{pdf}\left(\omega_{0}\right) d \omega_{0} \ldots \int_{4 \pi} \operatorname{pdf}\left(\omega_{m}\right) d \omega_{m} \ldots \\
& \left(W_{0, V S}+W_{1, V S}+W_{2, V S}+\cdots+W_{m, V S}+\cdots\right) \\
& W_{0, V S}=\frac{1}{p_{n} \quad \operatorname{pdf}(g) \operatorname{pdf}\left(V_{i}\right) \operatorname{pdf}\left(\omega_{0}\right)} \times \frac{\Gamma_{j} \kappa_{i} \tau_{i j} \varepsilon_{1} \Delta I_{i j}}{1} \\
& W_{1, V S}=\frac{1}{p_{n} \quad \operatorname{pdf}(g) \operatorname{pdf}\left(V_{i}\right) \operatorname{pdf}\left(\omega_{0}\right)} \times \frac{\Gamma_{j} \kappa_{i} \tau_{i j} \varepsilon_{2} \Delta I_{i j}\left(1-\varepsilon_{1}\right) \mathcal{F}_{1}\left(\mathbf{u}_{1} \mid \mathbf{u}_{0}\right)}{\operatorname{pdf}\left(\omega_{1}\right)} \\
& W_{2, V S}=\frac{1}{p_{n} \quad \operatorname{pdf}(g) \operatorname{pdf}\left(V_{i}\right) \operatorname{pdf}\left(\omega_{0}\right)} \\
& \times \frac{\Gamma_{j} \kappa_{i} \tau_{i j} \varepsilon_{3} \Delta I_{i j}\left(1-\varepsilon_{1}\right) \mathcal{F}_{1}\left(\mathbf{u}_{1} \mid \mathbf{u}_{0}\right)\left(1-\varepsilon_{2}\right) \mathcal{F}_{2}\left(\mathbf{u}_{2} \mid \mathbf{u}_{1}\right)}{\operatorname{pdf}\left(\omega_{1}\right) \operatorname{pdf}\left(\omega_{2}\right)} \\
& W_{m, V S}=\frac{1}{p_{n} \quad \operatorname{pdf}(g) \operatorname{pdf}\left(V_{i}\right) \operatorname{pdf}\left(\omega_{0}\right)} \\
& \times \frac{\left(1-\varepsilon_{1}\right) \mathcal{F}_{1}\left(\mathbf{u}_{1} \mid \mathbf{u}_{0}\right)\left(1-\varepsilon_{2}\right) \mathcal{F}_{2}\left(\mathbf{u}_{2} \mid \mathbf{u}_{1}\right) \cdots\left(1-\varepsilon_{m}\right) \mathcal{F}_{m}\left(\mathbf{u}_{m} \mid \mathbf{u}_{m-1}\right)}{\operatorname{pdf}\left(\omega_{1}\right) \operatorname{pdf}\left(\omega_{2}\right) \cdots \operatorname{pdf}\left(\omega_{m}\right)} \\
& \times \Gamma_{j} \kappa_{i} \tau_{i j} \varepsilon_{m+1} \Delta I_{i j} \\
& \varphi_{\left(S_{i}, V_{j}\right)}=\sum_{n=1}^{N_{b}} p_{n} \Delta_{v n} \int_{0}^{1} \operatorname{pdf}(g) d \kappa \int_{S_{i}} \operatorname{pdf}\left(S_{i}\right) d S_{i} \\
& \int_{4 \pi} \operatorname{pdf}\left(\omega_{0}\right) d \omega_{0} \int \operatorname{pdf}\left(\sigma_{0}\right) d \sigma_{0} \ldots \\
& \int_{4 \pi} \operatorname{pdf}\left(\omega_{0}\right) d \omega_{0} \int \operatorname{pdf}\left(\sigma_{m}\right) d \sigma_{m} \ldots \\
& \left(W_{0, S V}+W_{1, S V}+W_{2, S V}+\cdots+W_{m, S V}+\cdots\right) \\
& W_{0, S V}=\frac{1}{p_{n} \quad \operatorname{pdf}(g) \operatorname{pdf}\left(S_{i}\right) \operatorname{pdf}\left(\omega_{0}\right)} \times \frac{\Gamma_{j} \varepsilon_{i} \cos \theta_{i} \tau_{i j} \kappa_{j} \Delta I_{i j}}{\operatorname{pdf}\left(\sigma_{0}\right)}
\end{aligned}
$$




$$
\begin{aligned}
& W_{1, S V}=\frac{1}{p_{n} \quad \operatorname{pdf}(g) \operatorname{pdf}\left(S_{i}\right) \operatorname{pdf}\left(\omega_{0}\right)} \times \frac{\Gamma_{j} \varepsilon_{i} \cos \theta_{i} \tau_{i j} \kappa_{j} \Delta I_{i j}\left(1-\varepsilon_{1}\right) \mathcal{F}_{1}\left(\mathbf{u}_{1} \mid \mathbf{u}_{0}\right)}{\operatorname{pdf}\left(\sigma_{1}\right) \operatorname{pdf}\left(\omega_{1}\right)} \\
& W_{2, S V}=\frac{1}{p_{n} \quad \operatorname{pdf}(g) \operatorname{pdf}\left(S_{i}\right) \operatorname{pdf}\left(\omega_{0}\right)} \\
& \times \frac{\Gamma_{j} \varepsilon_{i} \cos \theta_{i} \tau_{i j} \kappa_{j} \Delta I_{i j}\left(1-\varepsilon_{1}\right) \mathcal{F}_{1}\left(\mathbf{u}_{1} \mid \mathbf{u}_{0}\right)\left(1-\varepsilon_{2}\right) \mathcal{F}_{2}\left(\mathbf{u}_{2} \mid \mathbf{u}_{1}\right)}{\operatorname{pdf}\left(\sigma_{2}\right) \operatorname{pdf}\left(\omega_{1}\right) \operatorname{pdf}\left(\omega_{2}\right)} \\
& W_{m, S V}=\frac{1}{p_{n} \quad \operatorname{pdf}(g) \operatorname{pdf}\left(S_{i}\right) \operatorname{pdf}\left(\omega_{0}\right)} \\
& \times \frac{\left(1-\varepsilon_{1}\right) \mathcal{F}_{1}\left(\mathbf{u}_{1} \mid \mathbf{u}_{0}\right)\left(1-\varepsilon_{2}\right) \mathcal{F}_{2}\left(\mathbf{u}_{2} \mid \mathbf{u}_{1}\right) \cdots\left(1-\varepsilon_{m}\right) \mathcal{F}_{m}\left(\mathbf{u}_{m} \mid \mathbf{u}_{m-1}\right)}{\operatorname{pdf}\left(\sigma_{m}\right) \operatorname{pdf}\left(\omega_{1}\right) \operatorname{pdf}\left(\omega_{2}\right) \cdots \operatorname{pdf}\left(\omega_{m}\right)} \\
& \times \Gamma_{j} \varepsilon_{i} \cos \theta_{i} \tau_{i j} \kappa_{j} \Delta I_{i j} \\
& \varphi_{\left(S_{i}, S_{j}\right)}=\sum_{n=1}^{N_{b}} p_{n} \Delta_{v n} \int_{0}^{1} \operatorname{pdf}(g) d \kappa \int_{S_{i}} \operatorname{pdf}\left(S_{i}\right) d S_{i} \\
& \int_{4 \pi} \operatorname{pdf}\left(\omega_{0}\right) d \omega_{0} \cdots \int_{4 \pi} \operatorname{pdf}\left(\omega_{m}\right) d \omega_{m} \cdots \\
& \left(W_{0, S S}+W_{1, S S}+W_{2, S S}+\cdots+W_{m, S S}+\cdots\right) \\
& W_{0, S S}=\frac{1}{p_{n} \quad \operatorname{pdf}(g) \operatorname{pdf}\left(S_{i}\right) \operatorname{pdf}\left(\omega_{0}\right)} \times \frac{\Gamma_{j} \varepsilon_{i} \cos \theta_{i} \tau_{i j} \varepsilon_{1} \Delta I_{i j}}{1}
\end{aligned}
$$$$
W_{1, S S}=\frac{1}{p_{n} \quad \operatorname{pdf}(g) \operatorname{pdf}\left(S_{i}\right) \operatorname{pdf}\left(\omega_{0}\right)} \times \frac{\Gamma_{j} \varepsilon_{i} \cos \theta_{i} \tau_{i j} \varepsilon_{2} \Delta I_{i j}\left(1-\varepsilon_{1}\right) \mathcal{F}_{1}\left(\mathbf{u}_{1} \mid \mathbf{u}_{0}\right)}{\operatorname{pdf}\left(\omega_{1}\right)}
$$$$
W_{2, S S}=\frac{1}{p_{n} \quad \operatorname{pdf}(\mathrm{g}) \operatorname{pdf}\left(\mathrm{S}_{\mathrm{i}}\right) \operatorname{pdf}\left(\omega_{0}\right)}
$$$$
\times \frac{\Gamma_{j} \varepsilon_{i} \cos \theta_{i} \tau_{i j} \varepsilon_{3} \Delta I_{i j}\left(1-\varepsilon_{1}\right) \mathcal{F}_{1}\left(\mathbf{u}_{1} \mid \mathbf{u}_{0}\right)\left(1-\varepsilon_{2}\right) \mathcal{F}_{2}\left(\mathbf{u}_{2} \mid \mathbf{u}_{1}\right)}{\operatorname{pdf}\left(\omega_{1}\right) \operatorname{pdf}\left(\omega_{2}\right)}
$$$$
W_{m, S S}=\frac{f(\kappa)}{p_{n} \quad \operatorname{pdf}(\kappa) \operatorname{pdf}\left(\mathrm{S}_{\mathrm{i}}\right) \operatorname{pdf}\left(\omega_{0}\right)}
$$$$
\times \frac{\left(1-\varepsilon_{1}\right) \mathcal{F}_{1}\left(u_{1} \mid \mathbf{u}_{0}\right)\left(1-\varepsilon_{2}\right) \mathcal{F}_{2}\left(u_{2} \mid u_{1}\right) \cdots\left(1-\varepsilon_{m}\right) \mathcal{F}_{m}\left(u_{m} \mid u_{m-1}\right)}{\operatorname{pdf}\left(\omega_{1}\right) \operatorname{pdf}\left(\omega_{2}\right) \ldots \operatorname{pdf}\left(\omega_{m}\right)}
$$$$
\times \Gamma_{j} \varepsilon_{i} \cos \theta_{i} \tau_{i j} \varepsilon_{m+1} \Delta I_{i j}
$$ 


\section{APPENDIX B: NUMERICAL RESULTS FOR TEST CASE 1 AND TEST CASE 2}

Table 1. Numerical results for test case 1: $\mathrm{q}_{w}$ is the flux on the lateral wall of the cylinder, and $\nabla \cdot \mathbf{q}$ is the radiative source term on the axis of the cylinder. Data from the MCM and associated statistical errors

\begin{tabular}{llllll}
\hline$x(\mathrm{~m})$ & $\nabla \cdot q\left(\mathrm{~kW} / \mathrm{m}^{3}\right)$ & $q_{w}\left(\mathrm{~kW} / \mathrm{m}^{2}\right)$ & $x(\mathrm{~m})$ & $\nabla \cdot q\left(\mathrm{~kW} / \mathrm{m}^{3}\right)$ & $q_{w}\left(\mathrm{~kW} / \mathrm{m}^{2}\right)$ \\
\hline 0.0205 & $770.83 \pm 1.58$ & $232.07 \pm 0.39$ & 0.6505 & $513.84 \pm 1.54$ & $300.06 \pm 0.48$ \\
0.0405 & $697.80 \pm 1.47$ & $242.02 \pm 0.41$ & 0.7005 & $511.13 \pm 1.53$ & $300.84 \pm 0.51$ \\
0.0605 & $657.24 \pm 1.45$ & $249.33 \pm 0.41$ & 0.7505 & $509.64 \pm 1.55$ & $302.39 \pm 0.52$ \\
0.0805 & $632.61 \pm 1.45$ & $254.26 \pm 0.40$ & 0.8005 & $509.40 \pm 1.51$ & $302.18 \pm 0.51$ \\
0.1005 & $614.27 \pm 1.41$ & $259.22 \pm 0.42$ & 0.8505 & $506.26 \pm 1.48$ & $303.88 \pm 0.51$ \\
0.1205 & $601.34 \pm 1.38$ & $262.94 \pm 0.37$ & 0.9005 & $505.15 \pm 1.45$ & $304.63 \pm 0.53$ \\
0.1405 & $589.66 \pm 1.52$ & $266.72 \pm 0.44$ & 0.9505 & $503.50 \pm 1.45$ & $305.38 \pm 0.49$ \\
0.1605 & $582.51 \pm 1.53$ & $269.55 \pm 0.47$ & 1.0005 & $502.54 \pm 1.49$ & $305.67 \pm 0.52$ \\
0.1805 & $575.40 \pm 1.59$ & $272.38 \pm 0.46$ & 1.0505 & $501.63 \pm 1.49$ & $306.21 \pm 0.55$ \\
0.2005 & $568.26 \pm 1.53$ & $274.10 \pm 0.48$ & 1.1005 & $501.46 \pm 1.48$ & $306.69 \pm 0.53$ \\
0.2505 & $555.22 \pm 1.47$ & $279.82 \pm 0.45$ & 1.1505 & $501.01 \pm 1.56$ & $307.34 \pm 0.52$ \\
0.3005 & $547.66 \pm 1.47$ & $283.67 \pm 0.42$ & 1.2005 & $499.73 \pm 1.45$ & $307.88 \pm 0.51$ \\
0.3505 & $537.50 \pm 1.57$ & $287.61 \pm 0.47$ & 1.2505 & $499.61 \pm 1.64$ & $307.85 \pm 0.49$ \\
0.4005 & $531.34 \pm 1.55$ & $290.29 \pm 0.49$ & 1.3005 & $499.44 \pm 1.64$ & $307.10 \pm 0.51$ \\
0.4505 & $528.04 \pm 1.57$ & $292.47 \pm 0.51$ & 1.3505 & $499.51 \pm 1.63$ & $308.10 \pm 0.54$ \\
0.5005 & $523.93 \pm 1.56$ & $295.15 \pm 0.51$ & 1.4005 & $499.56 \pm 1.77$ & $308.39 \pm 0.53$ \\
0.5505 & $519.16 \pm 1.51$ & $296.60 \pm 0.51$ & 1.4505 & $496.62 \pm 1.64$ & $308.12 \pm 0.52$ \\
0.6005 & $516.65 \pm 1.56$ & $298.08 \pm 0.47$ & 1.4905 & $498.89 \pm 1.59$ & $307.91 \pm 0.49$ \\
\hline
\end{tabular}

Table 2. Numerical results for test case 2: $\mathrm{q}_{w}$ is the net flux on the lateral wall of the cylinder, and $\nabla \cdot \mathbf{q}$ is the radiative source term on the axis of the cylinder. Data from the MCM and associated statistical errors

\begin{tabular}{lrrrrr}
\hline$x(\mathrm{~m})$ & $\nabla \cdot \mathbf{q}\left(\mathrm{kW} / \mathrm{m}^{3}\right)$ & $q_{w}\left(\mathrm{~kW} / \mathrm{m}^{2}\right)$ & $x(\mathrm{~m})$ & $\nabla \cdot \mathbf{q}\left(\mathrm{kW} / \mathrm{m}^{3}\right)$ & $q_{w}\left(\mathrm{~kW} / \mathrm{m}^{2}\right)$ \\
\hline 0.025 & $-5.25 \pm 1.05$ & $2.20 \pm 0.03$ & 0.625 & $1,281.18 \pm 11.63$ & $20.15 \pm 0.15$ \\
0.075 & $29.63 \pm 1.34$ & $2.98 \pm 0.03$ & 0.675 & $1,539.15 \pm 13.54$ & $22.36 \pm 0.17$ \\
0.125 & $69.98 \pm 1.92$ & $3.92 \pm 0.04$ & 0.725 & $1,827.86 \pm 14.24$ & $24.54 \pm 0.18$ \\
0.175 & $119.22 \pm 2.45$ & $4.97 \pm 0.05$ & 0.775 & $2,162.77 \pm 15.56$ & $26.81 \pm 0.20$ \\
0.225 & $177.76 \pm 3.40$ & $6.17 \pm 0.06$ & 0.825 & $2,554.37 \pm 17.50$ & $28.94 \pm 0.22$ \\
0.275 & $250.26 \pm 3.75$ & $7.50 \pm 0.07$ & 0.875 & $2,990.06 \pm 18.55$ & $30.85 \pm 0.23$ \\
0.325 & $335.50 \pm 4.64$ & $8.98 \pm 0.08$ & 0.925 & $3,489.83 \pm 20.37$ & $32.47 \pm 0.25$ \\
0.375 & $435.91 \pm 5.95$ & $10.54 \pm 0.09$ & 0.975 & $4,065.79 \pm 22.18$ & $33.20 \pm 0.26$ \\
0.425 & $558.42 \pm 6.72$ & $12.24 \pm 0.10$ & 1.025 & $4,741.95 \pm 24.85$ & $33.07 \pm 0.26$ \\
0.475 & $699.10 \pm 7.44$ & $14.06 \pm 0.11$ & 1.075 & $5,549.90 \pm 28.30$ & $31.56 \pm 0.27$ \\
0.525 & $864.32 \pm 8.50$ & $16.05 \pm 0.12$ & 1.125 & $6,530.07 \pm 31.34$ & $27.80 \pm 0.95$ \\
0.575 & $1,062.02 \pm 10.19$ & $18.02 \pm 0.14$ & 1.175 & $7,992.95 \pm 3.18$ & $20.70 \pm 1.16$ \\
\hline
\end{tabular}


Table 3. Numerical results for test case $2: q_{w}$ is the net flux on the lateral wall of the cylinder, and $\nabla . q$ is the radiative source term on the axis of the cylinder. Results from the DOM calculations

\begin{tabular}{|c|c|c|c|c|}
\hline \multirow[b]{2}{*}{$x(\mathrm{~m})$} & \multicolumn{2}{|c|}{ DOM-S16 } & \multicolumn{2}{|c|}{ DOM-S8 } \\
\hline & $\nabla \cdot q\left(\mathrm{~kW} / \mathrm{m}^{3}\right)$ & $q_{w}\left(\mathrm{~kW} / \mathrm{m}^{2}\right)$ & $\nabla \cdot q\left(\mathrm{~kW} / \mathrm{m}^{3}\right)$ & $q_{w}\left(\mathrm{~kW} / \mathrm{m}^{2}\right)$ \\
\hline 0.075 & 28.28 & 3.03 & 19.19 & 2.67 \\
\hline 0.225 & 185.67 & 6.20 & 171.26 & 5.73 \\
\hline 0.375 & 438.25 & 10.48 & 435.96 & 9.9 \\
\hline 0.525 & 901.89 & 15.83 & 868.35 & 15.24 \\
\hline 0.675 & $1,587.00$ & 22.03 & $1,540.04$ & 21.61 \\
\hline 0.825 & $2,604.75$ & 28.46 & $2,544.99$ & 28.02 \\
\hline 0.975 & $4,090.58$ & 32.62 & $4,017.71$ & 30.93 \\
\hline 1.125 & $6,404.20$ & 27.88 & $6,328.39$ & 28.17 \\
\hline
\end{tabular}

\section{APPENDIX C: MONTE CARLO INTEGRATION PRINCIPLE AND STATISTICAL ERRORS}

The statistical errors appearing in Tables 1 and 2 are computed together with the solution according to the standard Monte Carlo integration method, whose principle is reviewed hereafter.

Let us consider the integral $I=\int_{D} f(x) d x$. One can always introduce an arbitrary probability density function $p$ defined and strictly positive on the integration domain $D$ and define $g(x)=f(x) / p(x)$ so that

$$
I=\int_{D} \frac{f(x)}{p(x)} p(x) d x=\int_{D} g(x) p(x) d x
$$

Let us now define a random variable $X$ distributed according to $p$; then $g(X)$ is also a random variable and $I$ is the expectation of $g(X)$, and $I$ will be estimated with $N$ samples of $g(X)$ :

$$
I=E[g(X)] \approx \frac{1}{N} \sum_{i=1}^{N} g\left(x_{i}\right)=\langle g(X)\rangle_{N} \quad\left(I=\lim _{N \rightarrow \infty}\langle g(X)\rangle_{N}\right)
$$

The standard deviation of the estimate is $\sigma\left(\langle g(X)\rangle_{N}\right)=(1 / \sqrt{N}) \sigma[g(X)]$, where $\sigma[g(X)]$ is the standard deviation of $g(X)$, and will be approximated as

$$
\sigma\left(\langle g(X)\rangle_{N}\right) \approx \frac{1}{\sqrt{N}} \sqrt{\left[\left\langle g(X)^{2}\right\rangle_{N}-\langle g(X)\rangle_{N}^{2}\right]}
$$

The relative errors reported in Tables 1 and 2 correspond to this standard deviation estimate divided by the integral estimate. 